\title{
A large-scale correlated study of linear optical absorption and low-lying excited states of polyacenes: Pariser-Parr-Pople Hamiltonian
}

\author{
Priya Sony and Alok Shukla \\ Physics Department, Indian Institute of Technology, Powai, Mumbai 400076, INDIA*
}

\begin{abstract}
In this paper we present large-scale correlated calculations of linear optical absorption spectrum of oligo-acenes containing up to seven benzene rings. For the calculations we used the Pariser-ParrPople (P-P-P) Hamiltonian, along with the configuration interaction (CI) technique at various levels such as the full CI (FCI), the quadruple CI (QCI) and multi-reference singles-doubles CI (MRSDCI). The role of Coulomb parameters used in the P-P-P Hamiltonian was examined by considering standard Ohno parameters, as well as a screened set of parameters. A detailed analysis of the many-body character of the important excited states contributing to the linear absorption has also been performed. The results of our calculations have been compared extensively with the theoretical work of other authors, as well as with the experiments.

PACS numbers: $78.30 . \mathrm{Jw}, 78.20 . \mathrm{Bh}, 42.65 .-\mathrm{k}$
\end{abstract}




\section{INTRODUCTION}

In last few decades, there has been intensive research in the field of optical properties of acenes. $\frac{1,2,3,4,5}{2}$ It is well known that with the increase in the number of benzene rings in the acenes, the HOMO to LUMO gap decreases, making the material more conducting. It is in fact believed that an infinite linear acene, i.e., the polyacene could be a metal,,$\frac{4}{-}$ and at low temperatures, a superconductor $\stackrel{\underline{5}}{\underline{T}}$ Thus, higher acenes can prove to be potential candidates for preparing both optical and electronic devices. Polyacenes generally crystallize in well-defined structures, and their crystalline forms have found applications in novel optoelectronic devices such as light-emitting field-effect transistors. ${ }^{6}$ Three-dimensional structures of polyacenes have also been investigated theoretically for their electronic structure, transport and optical properties by several authors.., 8.9 Oligomers of polyacene such as naphthalene, anthracene, tetracene, pentacene, etc., are materials which are well-known for their interesting optical properties. ${ }^{10}$ Because of the high symmetry, they have separate optical response to radiation polarized along the conjugation direction, vis-a-vis the radiation polarized perpendicular to it. Several experimental investigations of linear optical properties of polyacenes have been performed over the years. These include linear absorp-

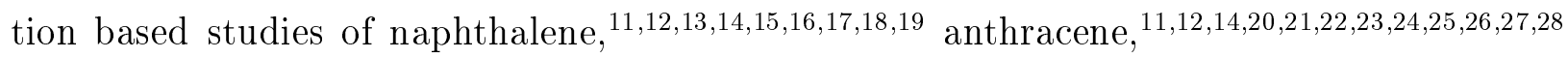

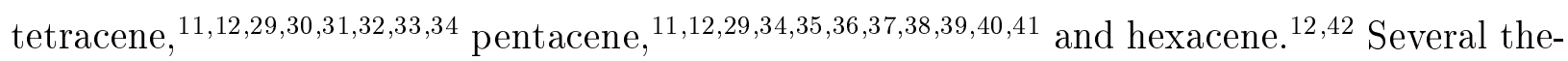
oretical studies of the low-lying excited states of these materials have also been performed, such as the early LCAO based study by Coulson, $\stackrel{43}{\rightarrow}$ LCAO-MO and perimeter MO approach by Pariser, $\underline{44}$ a free-electron-gas approach by Platt and Ham et al., $\stackrel{45,46}{ }$ CNDO/2 CI approach by Hofer et al., $\stackrel{47}{4}$ ab initio multi-reference Møller-Plesset (MRMP) theory ${ }^{48}$ density-matrix renormalization-group (DMRG) theory using P-P-P model Hamiltonian, $\underline{49.50}$ ab initio DFT based methodologies,, , $51,52,53, \underline{54}, 55$ self-consistent field-random phase approximation (SCFRPA) scheme using P-P-P model Hamiltonian, $\underline{56}$ and CNDO/S2 model parameterization technique by Lipari et al. $\frac{57}{}$ Among the more recent studies using the Pariser-Parr-Pople (PP-P) model Hamiltonian and many-body methodologies, of Ramasesha and coworkers $\underline{49}, \underline{50}$ are the foremost.

Most of the theoretical studies mentioned above concentrate either on a class of excited states of polyacenes, or restrict themselves to the study of smaller oligomers. In some earlier studies, chiefly because of the lack of computer power at the time, the level of treatment 
of electron-correlation effects was rather modest by contemporary standards. Additionally, to the best of our knowledge, none of the earlier theoretical studies reported calculations of optical absorption spectrum of these materials. Therefore, we believe that there is a need to perform systematic large-scale many-body calculations of optical properties of these materials with the following aims in mind: (i) to compute the linear optical absorption spectra of a range of oligoacenes, (ii) to understand their evolution with increasing size of the oligomers, (iii) to understand the influence of electron correlation effects on them, and finally (iv) to understand the nature of low-lying excited states contributing to the linear optics. As it is well-known, in quasi-one-dimensional materials such as conjugated polymers, electron-correlation effects have profound influence on their optical properties $\frac{58}{5}$ It is with all these issues in mind, in the present work, in a systematic manner, we undertake a large-scale correlated study of linear optical absorption in oligoacenes of increasing sizes, namely from naphthalene to heptacene. The optical absorption spectra have been computed both for the radiation polarized along the conjugation direction as well the one polarized perpendicular to it. We have used the P-P-P Hamiltonian for the purpose, and utilized various configuration interaction (CI) techniques such as the full CI (FCI), the quadruple CI (QCI), and the multireference singles-doubles CI (MRSDCI) methods. The CI-based correlation methodology used in the present work is sound, and has been used successfully by us in the past to study the linear and nonlinear optical properties of various other conjugated polymers $\underline{59}, \underline{60}, 61,62,63,64$ Additionally, we have also examined the issue of the influence of Coulomb parameters on the results by performing calculations with two distinct sets of parameters, namely the standard Ohno parameters $\frac{65}{5}$ and a screened set of parameters meant for phenylene-based conjugated polymers,$\underline{66}$ to describe the P-P-P model Hamiltonian.

The remainder of this paper is organized as follows. In section II we briefly review the theoretical methodology adopted in this work. In section III we present and discuss our results of the optical absorption spectra of various oligoacenes. Finally, in section IV we summarize our conclusions, and present possible directions for the future research work.

\section{THEORY}

The structures of oligoacenes $\left(\mathrm{C}_{4 n+2} \mathrm{H}_{2 n+4}, n=2,3,4,5,6\right.$, and 7$)$ starting from naphthalene up to heptacene are shown in Fig:1. Oligomers were assumed to lie in the $x y$-plane 
with the conjugation direction taken to be along the $x$-axis. They can be seen as a series of benzene rings fused together, along the conjugation direction. An alternative way to look at the structure of oligoacenes is to visualize them as two vertically displaced polyene chains coupled with each other along the $y$-axis via hopping, and the Coulomb interactions. From this viewpoint, polyacene is a ladder like polymer. The point group symmetry of oligoacenes is $D_{2 h}$, so that the one-photon states belong to the irreducible representations (irreps) $B_{3 u}$ or $B_{2 u}$, while the ground state belongs to the irrep $A_{g}$. Also, by convention we assign the ground state a negative (-) particle-hole symmetry. Therefore, by dipole selection rules, all the optically allowed states must have positive $(+)$ particle-hole symmetry. Thus, the states with negative particle-hole symmetry will not be visible in the linear optical spectrum. However, we have calculated the energy of $1 B_{3 u}^{-}$state, together with the optically allowed $1 B_{2 u}^{+}$and $1 B_{3 u}^{+}$states for the sake of comparison with the experimental and other theoretical results. Clar classified the absorption spectra into three bands, namely, $p, \alpha$, and $\beta . \underline{10}$ In our work, transition to $1 B_{2 u}^{+}$state from the $1 A_{g}^{-}$ground state via a short-axis (y-axis) polarized photon corresponds to the $p$ band $\left({ }^{1} L_{a}\right.$ band of Platt $\left.{ }^{45}\right)$. Similarly, transition from the ground state to $1 B_{3 u}^{-}$and $1 B_{3 u}^{+}$via a long-axis (x-axis) polarized photon corresponds to the $\alpha\left({ }^{1} L_{b}\right.$ band of Platt $\left.{ }^{45}\right)$ and the $\beta\left({ }^{1} B_{b}\right.$ band of Platt $\left.{ }^{45}\right)$ bands, respectively.

The correlated calculations on the oligoacenes were performed using the P-P-P model Hamiltonian, which can be written as

$$
H=H_{C_{1}}+H_{C_{2}}+H_{C_{1} C_{2}}+H_{e e}
$$

where $H_{C_{1}}$ and $H_{C_{2}}$ are the one-electron Hamiltonians for the carbon atoms located on the upper and the lower polyene chains, respectively. $H_{C_{1} C_{2}}$ is the one-electron hopping between the two chains, and $\mathrm{H}_{e e}$ depicts the electron-electron repulsion. The individual terms can now be written as,

$$
\begin{aligned}
& H_{C_{1}}=-t_{0} \sum_{\left\langle k, k^{\prime}\right\rangle} B_{k, k^{\prime}} \\
& H_{C_{2}}=-t_{0} \sum_{\langle\mu, \nu\rangle} B_{\mu, \nu}
\end{aligned}
$$

and 


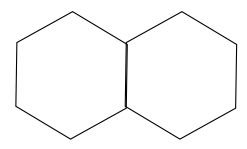

(a)

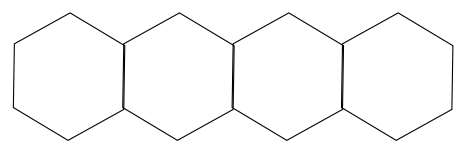

(c)

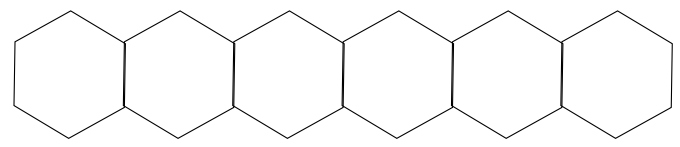

(e)

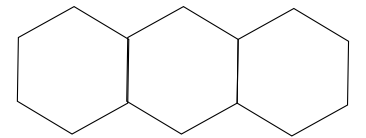

(b)

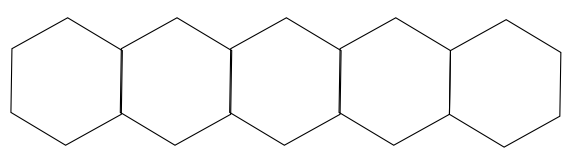

(d)

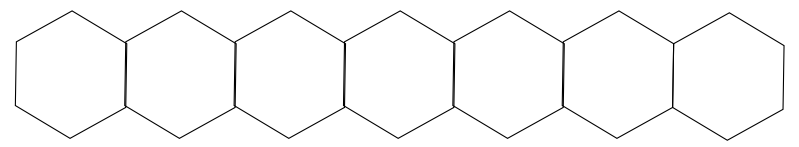

(f)

Figure 1: Schematic drawings of polyacenes considered in this work, namely, (a) naphthalene, (b) anthracene, (c) tetracene, (d) pentacene, (e) hexacene, and (f) heptacene

$$
\begin{gathered}
H_{C_{1} C_{2}}=-t_{\perp} \sum_{\langle k, \mu\rangle} B_{k, \mu} . \\
H_{e e}=U \sum_{i} n_{i \uparrow} n_{i \downarrow}+\frac{1}{2} \sum_{i \neq j} V_{i, j}\left(n_{i}-1\right)\left(n_{j}-1\right)
\end{gathered}
$$

In the equation above, $k, k^{\prime}$ are carbon atoms on the upper polyene chain, $\mu, \nu$ are carbon atoms located on the lower polyene chain, while $i$ and $j$ represent all the atoms of the oligomer. Symbol $\langle\ldots\rangle$ implies nearest neighbors, and $B_{i, j}=\sum_{\sigma}\left(c_{i, \sigma}^{\dagger} c_{j, \sigma}+\right.$ h.c. $)$. Matrix elements $t_{0}$, and $t_{\perp}$ depict one-electron hops. As far as the values of the hopping matrix elements are concerned, we took $t_{0}=2.4 \mathrm{eV}$ for both intracell and intercell hopping, and $t_{\perp}=t_{0}$ consistent with the undimerized ground state for polyacene argued by Raghu et al. $\underline{\underline{49}}$ Consequently, the carbon-carbon bond length has been fixed at $1.4 \AA$, and all bond angles have been taken to be $120^{\circ}$. At this point it is worthwhile to discuss the issue of the ground state geometry of oligoacenes. Experimentally speaking, to the best of our knowledge, the available data on the ground state geometry of various polyacenes is for the crystalline phase. ${ }^{67}$ Therefore, as far as the ground state geometries of isolated chains are concerned, 
theoretical calculations based upon geometry optimization provide very important input. However, the picture which emerges from such calculations is far from clear. Raghu et $a l . \stackrel{49}{n}$ studied the ground-state geometry of polyacenes using a DMRG based approach and concluded that the Peierls' instability in this polymer is conditional, with the gain in the electronic energy being proportional to $\delta^{2}$ ( $\delta$ is the dimerization amplitude), rather than $\delta$ which is the case for trans-polyacetylene. In their next paper, Raghu et $a l^{50}$ concluded that the ground state geometry of polyacene consists of a weakly distorted structure with undimerized chains, coupled by slightly longer rungs. Therefore, Raghu et al. ${ }^{50}$ used an undistorted geometry in their excited state calculations. Houk et al. $\frac{55}{}$, based upon their ab initio DFT based calculations, had also predicted a ground state similar to that of Raghu et al. $\underline{\underline{50}}$ Several workers have suggested that due to Peierls distortion acenes possess nonsymmetric geometry, ${ }^{51,53,68,69}$ but on the other hand Cioslowski ${ }^{70}$ has reported that at the correlated level, the symmetric geometry is more stable. Also, Klein and coworkers ${ }^{71}$ studied $^{2}$ the distortion of polyacenes within a many-body valence-bond framework, have suggested that all the structures (symmetric or nonsymmetric) are close in energy with symmetric one possessing the lowest energy. Moreover, Niehaus et al. $\stackrel{72}{ }$ have recently studied polyacenes using a tight-binding-based Green's-function approach, and reported that for $n \leq 19$, the symmetric structure is the most stable. Therefore, keeping these uncertainties in mind, and the fact that our main aim is to study the influence of electron correlations on the optical properties of oligoacenes, we have performed our calculations using the symmetrical structure for oligoacenes. This choice also allows us to compare our results directly with those of Ramasesha and coworkers, $\stackrel{50,73,74}{\underline{7}}$ wo also employed a symmetric geometry in their excited state of oligoacenes using P-P-P model Hamiltonian.

The Coulomb interactions are parameterized according to the Ohno relationship,$\underline{65}$

$$
V_{i, j}=U / \kappa_{i, j}\left(1+0.6117 R_{i, j}^{2}\right)^{1 / 2}
$$

where, $\kappa_{i, j}$ depicts the dielectric constant of the system which can simulate the effects of screening, $U$ is the on-site repulsion term, and $R_{i, j}$ is the distance in $\AA$ between the $i$-th carbon and the the $j$-th carbon. In the present work, we have performed calculations with two parameter sets: (a) "standard parameters" with $U=11.13 \mathrm{eV}$ and $\kappa_{i, j}=1.0$, and (b) "screened parameters" with $U=8.0 \mathrm{eV}$ and $\kappa_{i, j}=2.0(i \neq j)$ and $\kappa_{i, i}=1$, proposed initially by Chandross and Mazumdar to study phenyl-based conjugated polymers. ${ }^{66}$ In our earlier 
studies of phenyl-based conjugated polymers such as the PDPA, PPP, and PPV etc., we found that the screened parameters generally provided much better of description of their optical properties as compared to the standard ones. $\underline{59,60,61,62,63,64}$

The starting point of the correlated calculations for various oligomers were the restricted Hartree-Fock (HF) calculations, using the P-P-P Hamiltonian. The many-body effects beyond HF were computed using different levels of the configuration interaction (CI) method, namely, full-CI (FCI), quadruples-CI (QCI), and the multi-reference singles-doubles CI (MRSDCI). Details of these CI-based many-body procedures have been presented in our earlier works. $\underline{59}, \underline{60}, 62,63$ From the CI calculations, we obtain the eigenfunctions and eigenvalues corresponding to the correlated ground and excited states of various oligomers. The many-body wave functions are used to compute the matrix elements of the dipole operator connecting the ground state to various excited states. These dipole matrix elements are in turn used to calculate the linear optical absorption spectra of various polyacenes.

\section{CALCULATIONS AND RESULTS}

In this section, first we will briefly discuss the main features of linear optical spectra of polyacenes computed within the independent-electron Hückel model. Next we will present and discuss the main results of this work, namely, the correlated linear absorption spectra of oligoacenes of increasing sizes, and compare our results with the other available experimental and theoretical results. A preliminary description of results for tetracene and pentacene, was

presented in an earlier work ${ }^{75}$. However, the results presented here are based upon more extensive calculations, and, therefore, they supersede our earlier results $\frac{75}{}$.

\section{A. Hückel Model Results}

Here we briefly discuss the salient features of the linear absorption spectra of oligoacenes computed using the tight-binding Hückel model, and presented in Fig.2. 

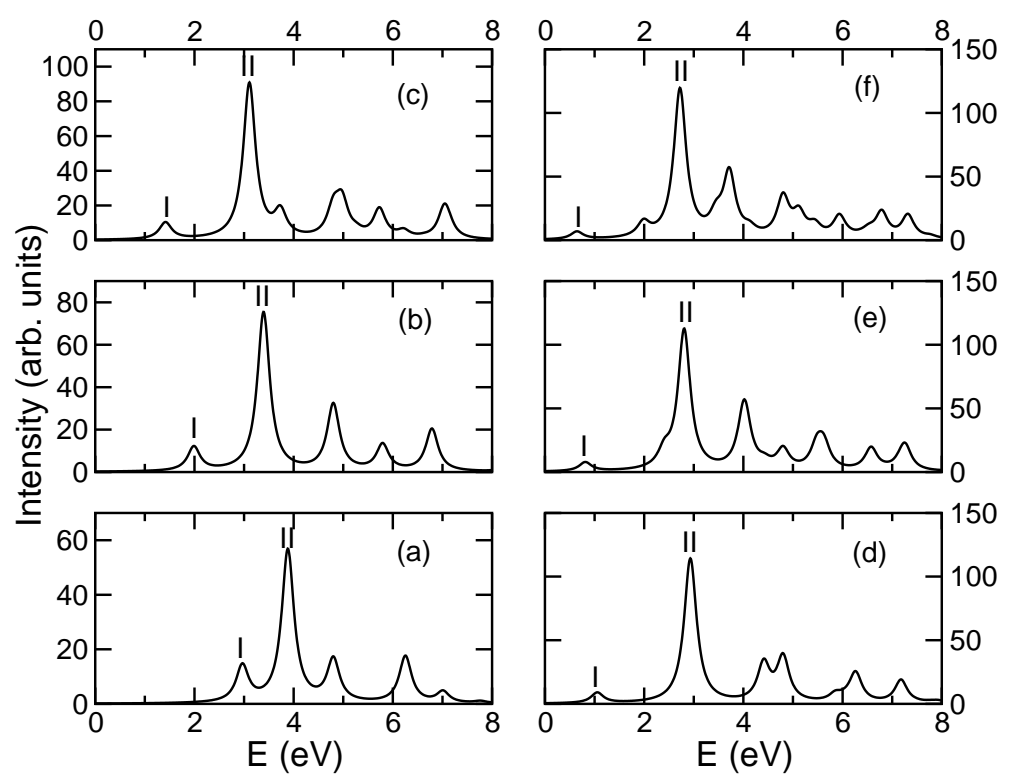

Figure 2: Linear optical absorption spectra of (a) naphthalene, (b) anthracene, (c) tetracene, (d) pentacene, (e) hexacene, and (f) heptacene. In all the cases a line width of $0.15 \mathrm{eV}$ was assumed.

For all the oligomers, the first peak (labeled I) corresponds to $\pi-\pi^{*}$ excitation described by HOMO $(H) \rightarrow$ LUMO $(L)$ transition through a $y$-polarized photon leading to the $1 B_{2 u}^{+}$ excited state of the system. However, with the increasing size of the oligomer, the intensity of this $H \rightarrow L$ transition decreases, which is understandable because, in the thermodynamic limit, with the chosen hopping parameters, the polyacene is metallic. The highest intensity peak (labeled II) for each of the oligoacene investigated corresponds to an $x$-polarized photon leading the system to its $1 B_{3 u}^{+}$excited state. For oligomers with $n=$ even, the highest intensity peak corresponds to the transitions $H \rightarrow L+n / 2$ and $H-n / 2 \rightarrow L$, while for those with $n=$ odd, the peak II corresponds to transitions $H \rightarrow L+(n \pm 1) / 2$ and $H-(n \pm 1) / 2 \rightarrow L$. Moreover, as the lengths of the oligoacenes increase, as expected, the spectrum is red shifted due to delocalization of particle-hole pair. 
Table I: The number of reference configurations $\left(N_{r e f}\right)$ and the total number of configurations $\left(N_{\text {total }}\right)$ involved in the MRSDCI (or FCI or QCI, where indicated) calculations, for different symmetry sub-spaces of various oligoacenes.

\begin{tabular}{|c|cc|cc|cc|}
\hline Oligomer & \multicolumn{2}{|c|}{$A_{g}$} & \multicolumn{2}{c|}{$B_{2 u}$} & \multicolumn{2}{c|}{$B_{3 u}$} \\
& $N_{\text {ref }}$ & $N_{\text {total }}$ & $N_{\text {ref }}$ & $N_{\text {total }}$ & $N_{\text {ref }}$ & $N_{\text {total }}$ \\
\hline naphthalene & $1^{a}$ & $4936^{a}$ & $1^{a}$ & $4794^{a}$ & $1^{a}$ & $4816^{a}$ \\
\hline anthracene & $1^{a}$ & $623576^{a}$ & $1^{a}$ & $618478^{a}$ & $1^{a}$ & $620928^{a}$ \\
\hline tetracene & $1^{b}$ & $193538^{b}$ & $1^{b}$ & $335325^{b}$ & $24^{c, d}$ & $34788^{c, d}$ \\
\hline pentacene & $1^{b}$ & $1002597^{b}$ & $1^{b}$ & $1707243^{b}$ & $38^{c}$ & $130196^{c}$ \\
& & & & & $34^{d}$ & $126690^{d}$ \\
\hline hexacene & $66^{c}$ & $460527^{c}$ & $28^{c}$ & $191944^{c}$ & $54^{c}$ & $393248^{c}$ \\
& $40^{d}$ & $299141^{d}$ & $32^{d}$ & $242013^{d}$ & $30^{d}$ & $252420^{d}$ \\
\hline heptacene & $45^{c}$ & $590599^{c}$ & $30^{c}$ & $415999^{c}$ & $40^{c, d}$ & $653476^{c, d}$ \\
& $36^{d}$ & $480032^{d}$ & $22^{d}$ & $270391^{d}$ & & \\
\hline
\end{tabular}

${ }^{a}$ FCI method with standard as well as screened parameters,

${ }^{b} \mathrm{QCI}$ method with standard as well as screened parameters,

${ }^{c}$ using standard parameters,

${ }^{d}$ using screened parameters.

\section{B. P-P-P Calculations}

Here we present the results of our correlated calculations of linear absorption on oligoacenes using the P-P-P model Hamiltonian. First we present and discuss our results for individual acenes, followed by a unified discussion of their spectra. In Table【 we present the number of reference states $\left(N_{\text {ref }}\right)$ and the dimension of the Hamiltonian matrix $\left(N_{\text {total }}\right)$ used in our CI calculations for different symmetry sub-spaces of various oligomers. The fact that the calculations presented here are quite large-scale is obvious from $N_{\text {total }}$, which, e.g., for pentacene was in excess of one million for the $A_{g}$ and $B_{2 u}$ symmetries. Therefore, we are confident that our results take into account the influence of electron-correlation effects quite accurately.

Before discussing the individual oligomers, we would like to summarize the patterns which 
Table II: Comparison of results of our calculations performed with the standard (Std.) parameters and the screened (Scd.) parameters with other experimental and theoretical results for the three most important low-lying states. For reference 51 (Kadantsev et al.),

results quoted with the asterisk $(*)$ correspond to their CISD calculations, while those without it are their B3LYP results.

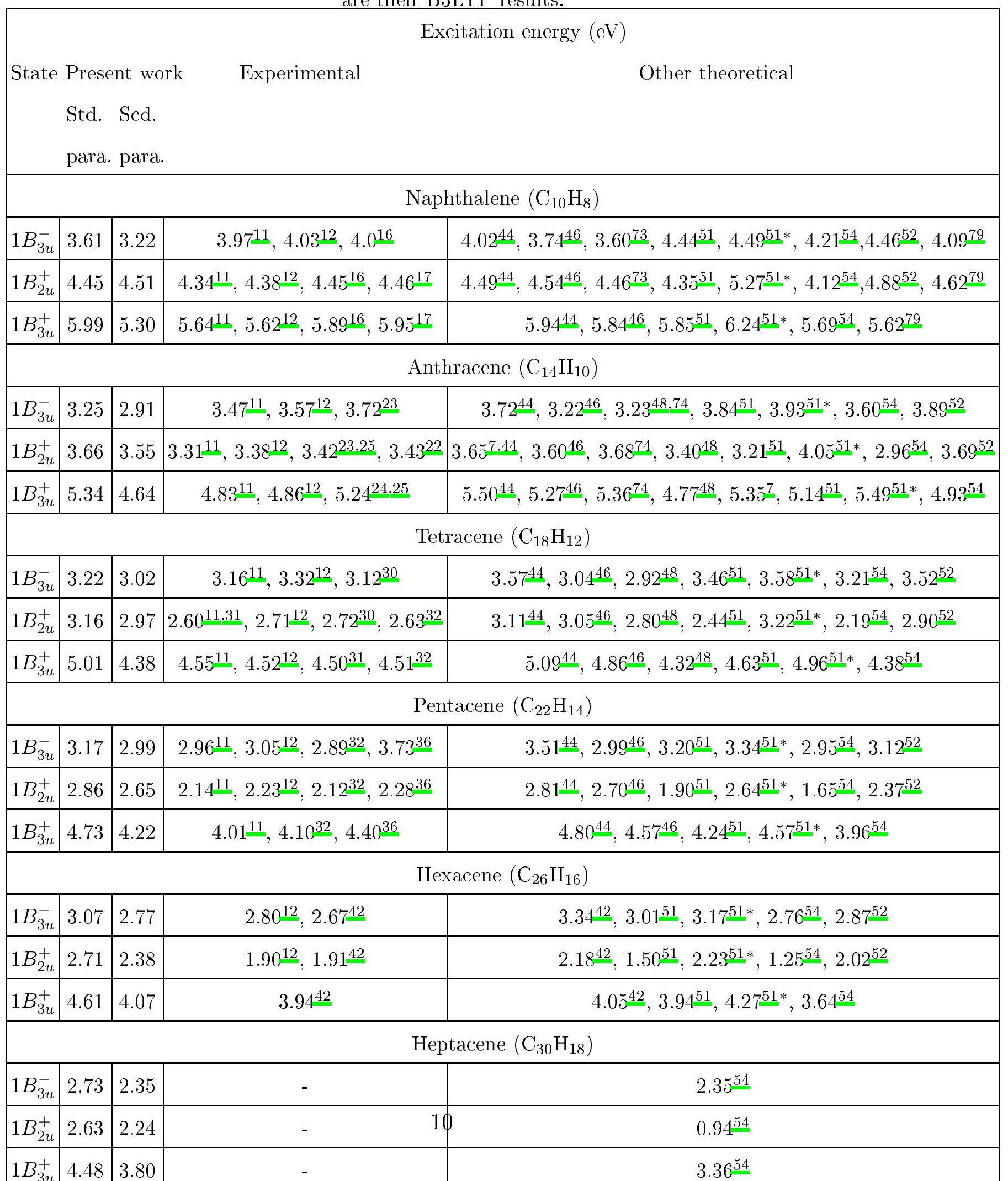


emerge from the computed optical absorption spectra of acenes ranging from naphthalene to heptacene. The absorption spectrum of all the oligoacenes, irrespective of the P-P-P parameters used in the calculations, contains following important features:

1. The first peak in the absorption spectrum for all the oligomers studied corresponds to the $1 B_{2 u}^{+}$excited state of the system. The most important configuration contributing to the many-particle wave function of the state corresponds to the $|H \rightarrow L\rangle$ excitation, in agreement with Hückel model calculations. The relative intensity of this feature decreases with the increasing size of the oligomer, again in agreement with the Hückel model results.

2. The second, and the most intense feature in the spectrum corresponds to the $1 B_{3 u}^{+}$ state, obtained by the absorption of a $x$-polarized photon. The most important configurations contributing to the wave function of this state, for $n=$ even oligomers are excitations $|H \rightarrow L+n / 2\rangle$ and $|H-n / 2 \rightarrow L\rangle$, and for $n=$ odd, the excitations $|H \rightarrow L+(n \pm 1) / 2\rangle$ and $|H-(n \pm 1) / 2 \rightarrow L\rangle$. This aspect of the correlated spectrum is also in good agreement with the Hückel model results.

3. Another important state, namely $1 B_{3 u}^{-}$state exists for all oligoacenes. Because it has the same particle-hole symmetry (-) as the ground state, in P-P-P (and Hückel) calculations it does not contribute to the absorption spectrum. But many experiments report this state as a very weak feature in the absorption spectrum. In our calculations, for naphthalene and anthracene, this state occurs at energies lower than the $1 B_{2 u}^{+}$state, but for longer oligomers, it is at higher excitation energies than the $1 B_{2 u}^{+}$state. The important configurations contributing to the wave function of this state are the same as the ones contributing to $1 B_{3 u}^{+}$, except for their opposite relative signs, for all the oligomers up to pentacene. But, from hexacene onwards, it is the doubly excited configurations which contribute significantly to this state. Thus, it is the electroncorrelation effects which are responsible for its distinct location in the spectrum as compared to the $1 B_{3 u}^{+}$state.

4. Generally, the spectra computed with the standard parameters are in good qualitative agreement with those computed with the screened parameters. Quantitatively speaking, screened parameter spectra are redshifted as compared to the standard parameter 
ones, and are in better agreement with the experiments for longer acenes.

5. Although there are some qualitative similarities between the P-P-P and Hückel model results, quantitatively speaking the optical gaps obtained using the Hückel model are much smaller than their P-P-P and experimental counterparts.

Having emphasized the general features of our work, next we discuss the results of our calculations for individual oligomers, and compare them with the theoretical results of other authors, and the experimental ones. Quantitative aspects of our calculations are summarized in table II, which also reports some of the experimental and the theoretical results of other authors. Additionally, in Appendix A, we present detailed tables for individual oligomers containing the excitation energies and many-particle wave functions of important excited states, as well as corresponding transition dipoles.

\section{Naphthalene}

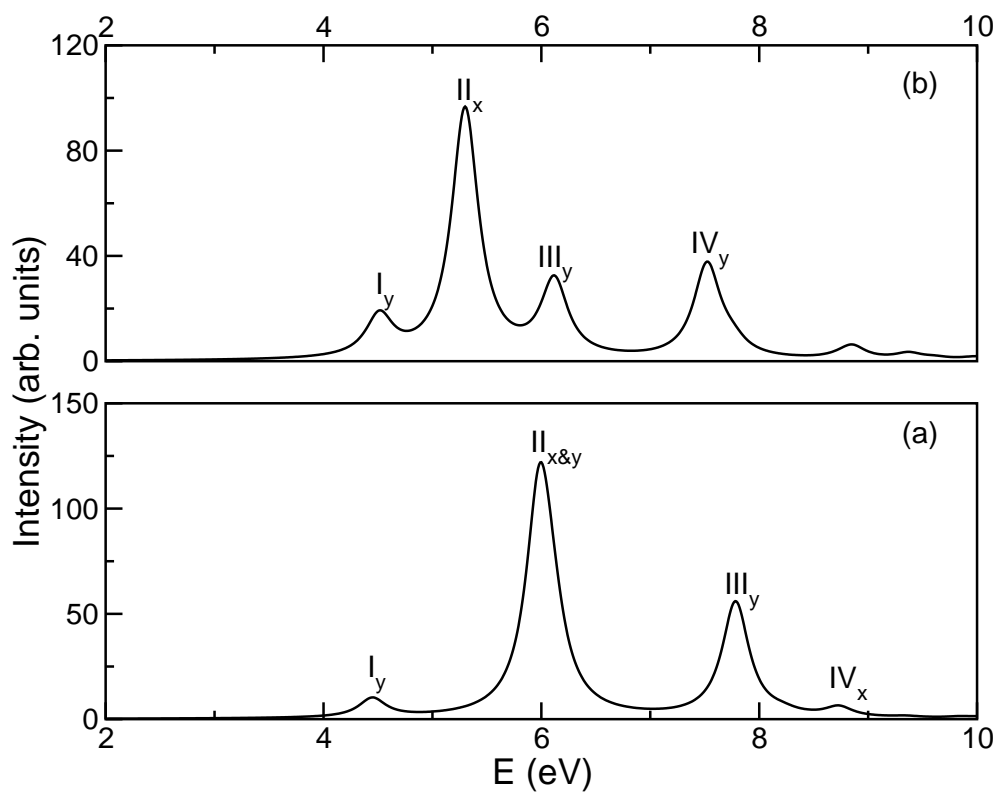

Figure 3: Linear optical absorption spectrum of naphthalene computed with (a) standard parameters and (b) screened parameters in the P-P-P model Hamiltonian. FCI method was used for the purpose. A line width of $0.15 \mathrm{eV}$ was assumed. As a subscript of each feature, its polarization is also mentioned. Thus, e.g., III $_{y}$ implies that peak III is short-axis polarized. 
In Fig 3 we present the linear optical absorption spectra of naphthalene computed with the FCI method, using both the standard and the screened parameters in the P-P-P Hamiltonian. The wave functions of the excited states contributing to various peaks are presented in Tables III and IV in the appendix. Since these calculations were performed using the FCI approach, they are exact within the model chosen, and cannot be improved. Therefore, any discrepancy which these results may exhibit with respect to the experiments, is a reflection of the limitations of the model or the parameters used, and not that of the correlation approach.

From Fig 3 and Tables III and IV, it is obvious that the first peak of the spectra calculated with the standard and screened parameters are in excellent qualitative and quantitative agreement with each other. The second peak computed using the standard parameters is due to both the $x$-polarized and the $y$-polarized components, but it is obvious from the Table III that $x$-polarized component dominates. However, with the screened parameters, it gets dissociated into two separate features II and III with $x$-polarized and $y$-polarized components, respectively. Qualitatively, both the spectra are in good agreement with each other (see Tables III and IV). Regarding the quantitative aspects, one notices that the excitation energies computed with the screened parameters are red shifted as compared to those computed with the standard parameters. This is in agreement with results obtained for other conjugated polymers as well $10,62,63,64$.

Comparison of our results for naphthalene, for the invisible (dipole forbidden) $1 B_{3 u}^{-}$state and the first two visible features, $1 B_{2 u}^{+}$, and $1 B_{3 u}^{+}$states with the experimental ones, and those of other calculations, is presented in Table II. Although, several other experimental results on optical absorption in naphthalene exist, $\underline{11,12,13,15,16,17,18,19}$ but here we compare our results with the experimental results of Huebner et al. $\frac{16}{}$ and Aleksandrovsky et al., $\frac{17}{1}$ who performed experiments on gas-phase of naphthalene. Since we have performed calculations on isolated oligomers, therefore, most appropriate comparison of our results will be with the data obtained by gas-phase experiments. Huebner et al..$^{16}$ suggested $1 B_{2 u}^{+}$and $1 B_{3 u}^{+}$states at $4.45 \mathrm{eV}$ and $5.89 \mathrm{eV}$ respectively, while Aleksandrovsky et al $\frac{17}{n}$ mentioned these states to be at $4.46 \mathrm{eV}$ and $5.95 \mathrm{eV}$ respectively. Thus, our results on the relative ordering of the $1 B_{2 u}^{+}$ and the $1 B_{3 u}^{+}$states in the spectra, using standard parameters are in excellent agreement with the results of Huebner et al. $\frac{16}{\underline{16}}$ and the recent experiment of Aleksandrovsky et al. $\underline{\underline{17}}$ The screened parameters slightly overestimate the experimental results $\frac{16.17}{17}$ for $1 B_{2 u}^{+}$state 
and underestimate them for $1 B_{3 u}^{+}$state. Excellent agreement between our results and those of recent experimental results of Aleksandrovsky et al. 17 testifies to the essential correctness of the P-P-P model to the naphthalene with standard parameters. The dipole forbidden $1 B_{3 u}^{-}$state has not been discussed by Aleksandrovsky et al. but Huebner et al. suggested this feature to be at $4.0 \mathrm{eV}$ which is higher as compared to both of our results. Thus, both sets of calculations appear to capture the qualitative features of the spectra quite well, however, quantitatively, standard parameter calculations appear to be more accurate upon comparison with the experiments $\underline{16}, \underline{17}$

Several theoreticians have studied the low-lying excited states of naphthalene $\underline{\underline{8}, 44,46,47,51,52,54,56,57,73,76,77,78,79}$ The results obtained from our standard set of parameters for the $1 B_{2 u}^{+}$and the $1 B_{3 u}^{+}$states are in perfect agreement with the calculations performed by Pariser et al $\underline{\underline{44}}$ and Ramasesha et al $\underline{\underline{73}}$ Also, they agree quite well with the recent calculations performed by Rubio and coworkers, $\frac{51}{1}$ using B3LYP as exchange-correlation (xc) functional. The dipole forbidden $1 B_{3 u}^{-}$state is obtained at lower energy than calculated by Pariser et al $\underline{\underline{44}}$ and Rubio and coworkers, $\underline{\underline{51}}$ but is in excellent agreement with the results of Ramasesha et al $\underline{\underline{73}}$

\section{Anthracene}

An anthracene molecule contains $14 \pi$-electrons, which is quite a large system for the application of the FCI approach. However, the FCI calculations using the P-P-P model have been performed by Ramasesha et al. $\underline{\underline{74}}$ for anthracene, but they did not report its linear absorption spectrum. Therefore, in our present work we have computed the linear absorption spectrum of the anthracene using the FCI approach, and have also discussed the nature of low-lying excited states contributing to the spectrum. 


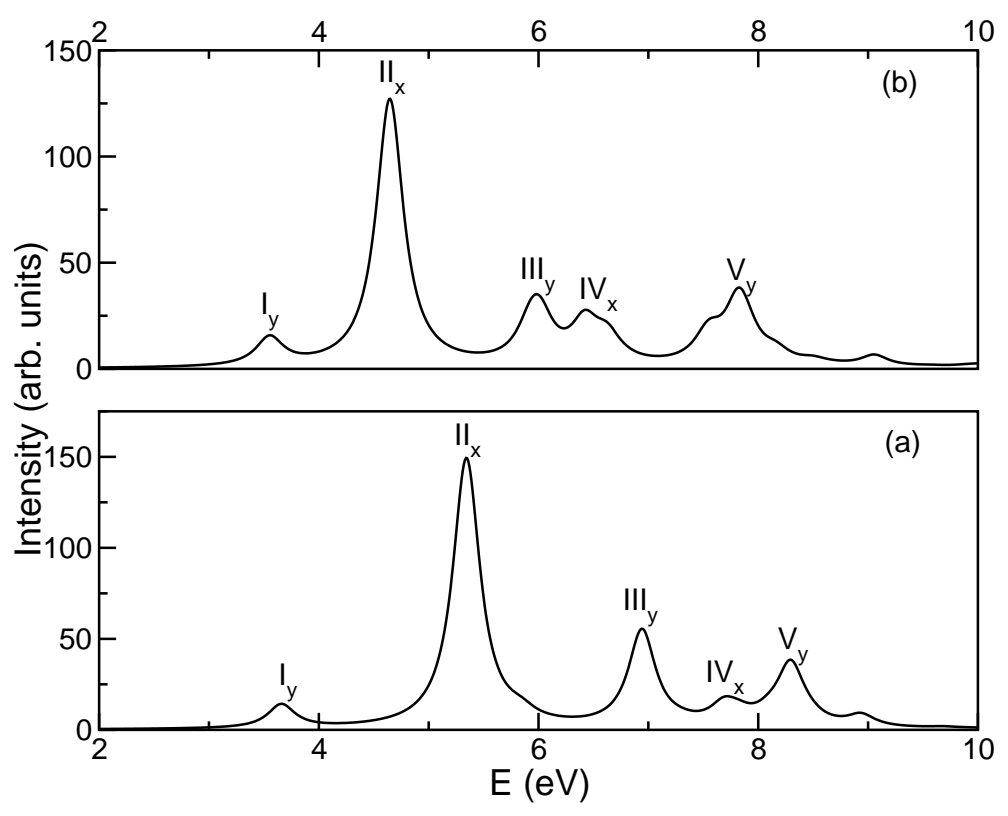

Figure 4: Linear optical absorption spectrum of anthracene computed with (a) standard parameters and (b) screened parameters. FCI method coupled with the P-P-P model Hamiltonian was used for the purpose. A line width of $0.15 \mathrm{eV}$ was assumed. As a subscript of each feature, its polarization is also mentioned.

Fig 4 presents the linear optical absorption spectra of anthracene computed using the FCI method, along with the standard and screened sets of parameters in the P-P-P Hamiltonian. The wave functions of the excited states contributing to various peaks are presented in Tables V and VI in the appendix.

In our calculations on anthracene using standard parameters, the first peak of the spectrum was identified as $1 B_{2 u}^{+}$state at $3.66 \mathrm{eV}$, while the most intense feature of the spectrum corresponding to the $1 B_{3 u}^{+}$state, was obtained at $5.34 \mathrm{eV}$. The dipole forbidden $1 B_{3 u}^{-}$state was obtained at $3.25 \mathrm{eV}$ and its wave function contains both singly and doubly excited configurations. Using screened parameters, we obtained $1 B_{3 u}^{-}, 1 B_{2 u}^{+}$, and $1 B_{3 u}^{+}$states at $2.91 \mathrm{eV}$, $3.55 \mathrm{eV}$, and $4.64 \mathrm{eV}$, respectively. Thus, calculations performed with both sets of P-P-P parameters predict the dipole forbidden $1 B_{3 u}^{-}$state at lower energies than the $1 B_{2 u}^{+}$state.

Comparison of our theoretical results, to the experimental and theoretical results of other authors, is presented in Table II. Several experimental investigations of the absorption spectra of anthracene have been performed over the years. For the $1 B_{2 u}^{+}$state, our results with both sets of parameters overestimate the experimental results of Klevens and Platt 
$(3.31 \mathrm{eV}), \underline{11}$, Biermann et al. $(3.38 \mathrm{eV}), \stackrel{12}{\underline{1}}$ Lambert et al. $(3.43 \mathrm{eV}), \stackrel{22}{ }$ Dick et al. $(3.42 \mathrm{eV}), \stackrel{23}{ }$ and Man et al. (3.424 eV) $\stackrel{25}{\underline{n}}$ Comparatively speaking, however, the result obtained from screened parameters is in slightly better agreement with the experimental results.

Lambert et $a l_{.}{ }^{22}$ studied the isolated molecules of anthracene using jet spectroscopy, while Dick et al. $\underline{23}$ observed the low-lying excited states of anthracene in gas-phase. Thus, our calculations, which were performed on isolated oligomers, are directly comparable to these two experimental results. The most intense feature of the spectra, which corresponds to $1 B_{3 u}^{+}$state was obtained at $5.34 \mathrm{eV}$ from standard parameters while screened parameters suggested this state to be at $4.64 \mathrm{eV}$. Our result with standard parameters agrees well with the results obtained by Man et al., $\frac{25}{2}$ and Lyons et al. ${ }^{24}$ They both suggested this state to be at $5.24 \mathrm{eV}$. Our screened parameter result is in good agreement with the result of Klevens and Platt, $\stackrel{11}{\underline{1}}$ and Biermann et al. $\stackrel{12}{\underline{n}}$ for the $1 B_{3 u}^{+}$state, who suggested this state to be at 4.83 $\mathrm{eV}$ and $4.86 \mathrm{eV}$, respectively.

Our correlated value of $3.25 \mathrm{eV}$ for the excitation energy of the $1 B_{3 u}^{-}$state obtained using the standard parameters is in fairly good agreement with the experimental results of Klevens and Platt $(3.47 \mathrm{eV})^{11}$ and Biermann et al. $(3.57 \mathrm{eV}) .12$ However, it is lower as compared to the experimental results of Dick et al. $(3.72 \mathrm{eV}){ }^{23}$ Our screened parameter results $(2.91$ $\mathrm{eV}$ ) obviously significantly underestimate the experimental excitation energy of the $1 B_{3 u}^{-}$ state. Thus, we observe that for some states our standard parameter results agree well with the experiments, while for other states screened parameter results are in better agreement with the experiments. However, the standard-parameter-based results are in better overall agreement with the gas-phase experiments, as compared to those obtained using screened parameters.

Next we discuss and compare our results with the results of calculations performed by other theoreticians. Our correlated results obtained by using standard parameters for $1 B_{3 u}^{-}$, $1 B_{2 u}^{+}$, and $1 B_{3 u}^{+}$states are in perfect agreement with the results obtained by Ramasesha et al.,$\underline{74}$ using FCI methodology with P-P-P model Hamiltonian. Again, this perfect agreement proves the essential correctness of our calculations. The $1 B_{2 u}^{+}$state obtained using standard parameters shows an excellent agreement with the pioneering work of Pariser, $\stackrel{44}{,}$ who performed the SCI calculations. However, energetically both $1 B_{3 u}^{+}$(dipole allowed) and $1 B_{3 u}^{-}$(dipole forbidden) states obtained from our calculations are lower than those reported by Pariser. $\stackrel{44}{ }$ This clearly is due to the superior treatment of electron correlations in our 
work. Additionally, the results for the $1 B_{2 u}^{+}$, and $1 B_{3 u}^{+}$states are in excellent agreement with results of Hummer et al. $\underline{7}^{\frac{7}{}}$ and in close agreement with the recent results of Rubio and coworkers, ${ }^{51}$ obtained using B3LYP as xc functional. Recently, Kawashima and co-workers ${ }^{48}$ performed calculations on linear absorption in anthracene using ab initio MRMP methodology. The energies they obtained for $1 B_{2 u}^{+}$state $(3.40 \mathrm{eV})$ and $1 B_{3 u}^{+}$state $(4.77 \mathrm{eV})$ are quite close to our screened parameter results, and slightly less than the results obtained from our calculations using standard parameters. They mentioned $1 B_{3 u}^{-}$state at $3.23 \mathrm{eV}$ which is in perfect agreement with the results which we have obtained using standard parameters, while it is higher than the value which we have obtained using screened parameters $(2.91$ $\mathrm{eV}$ ). Few other theoretical results of other authors are also given in the Table Ifor the sake of comparison.

\section{Tetr}

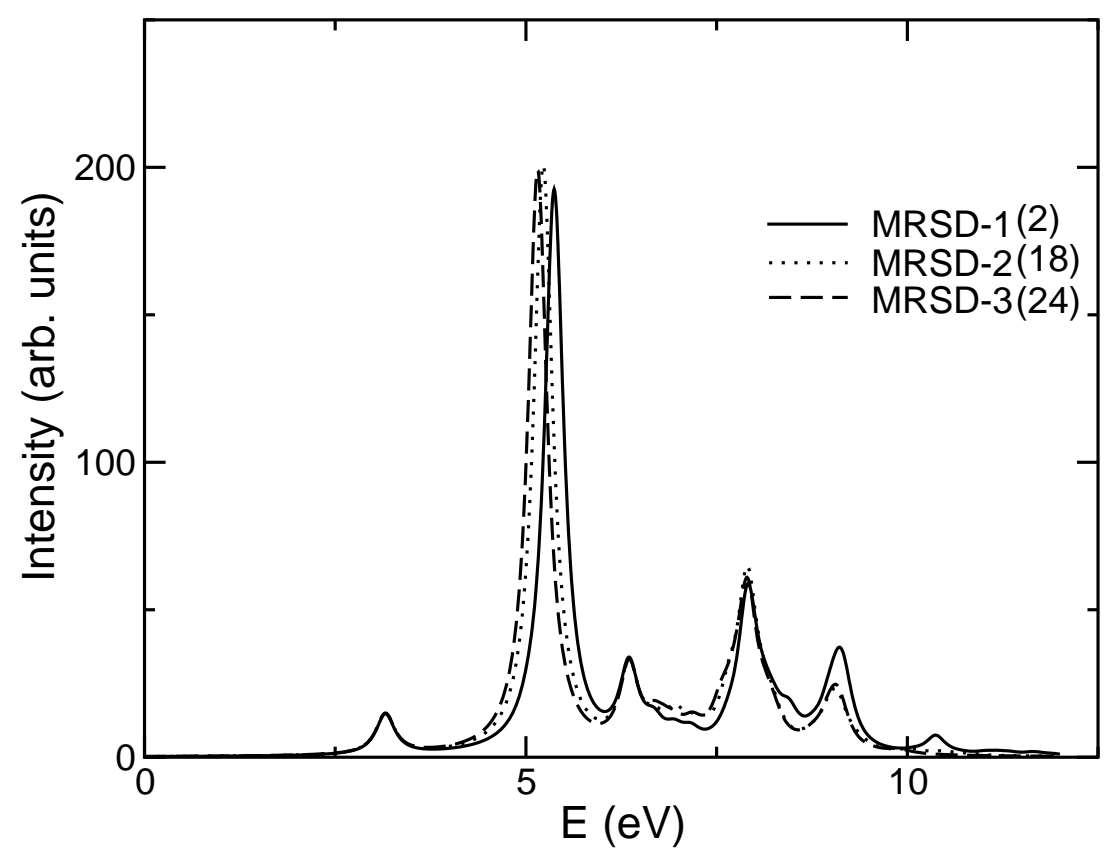

Figure 5: Convergence of linear absorption spectrum of tetracene, computed using the MRSDCI method (standard parameters) with respect to number of reference configurations $\left(N_{\text {ref }}\right)$. The numbers written in the parenthesis represent $N_{\text {ref }}$ included in the corresponding MRSDCI calculations. 
The next member of the polyacene family is tetracene, which contains $18 \pi$-electrons. Because of relatively larger number of $\pi$-electrons in the system, it is virtually impossible to use the FCI method for the present system. Thus, we used QCI technique for computing the ground state and the low-lying excited states corresponding to the short-axis polarized transitions ( $B_{2 u}$-type states), while MRSDCI methodology for the excited states which cor-

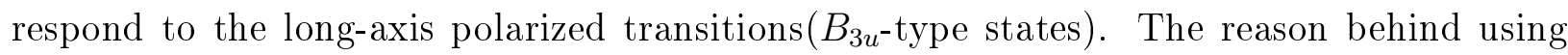
different CI techniques is that the QCI method can only be used for single reference states such as the ground state $\left(1 A_{g}\right)$ and the $B_{2 u}$ type states, while from previous Tables III, IV], $\mathrm{V}$, and VI, it is obvious that $B_{3 u}$ states are multi-reference states with two dominant configurations for which QCI method cannot be used. Therefore, for states of $B_{3 u}$ symmetry, the MRSDCI method has been used.

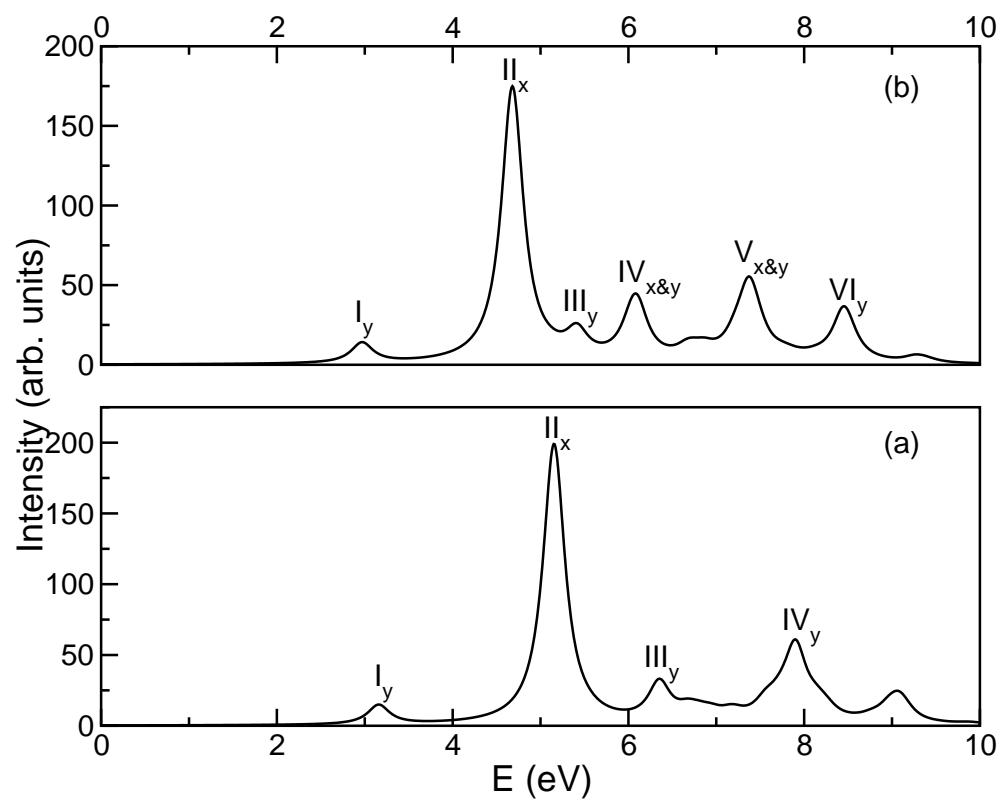

Figure 6: Linear optical absorption spectrum of tetracene computed with (a) standard parameters, and (b) screened parameters. QCI method was used to compute the ground state $\left(1 A_{g}^{-}\right.$state) and $B_{2 u}^{+}$states, while MRSDCI method was used to compute $B_{3 u}^{+}$states.

In order to demonstrate the convergence of our MRSDCI calculations, we present the spectra in Fig 5, calculated with increasing number of reference configurations $\left(N_{\text {ref }}\right)$. It is obvious from the figure that the spectrum computed using twenty four reference configurations is in very good agreement with the spectrum computed using eighteen reference configurations, implying that the convergence with respect to $N_{\text {ref }}$ has been achieved. We 
would also like to mention that the accuracy of the MRSDCI method was demonstrated in our earlier works where the results obtained using that approach were found to be in excellent agreement with the experiments. $\underline{59}, 60,61$

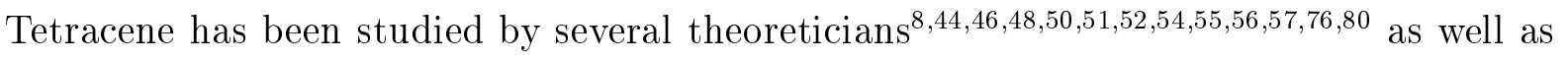
experimentalists. $11,12,29,30,31,32,33,34$ The linear absorption spectra of tetracene, computed using standard and screened parameters are presented in Fig.6. The excited states together with the corresponding energies, transition dipoles, and wave functions are presented in Tables VII and VIII.

The first peak of the correlated spectra was obtained at $3.16 \mathrm{eV}$ and $2.97 \mathrm{eV}$, using the standard parameters and the screened parameters, respectively, and corresponds to the $1 B_{2 u}^{+}$ state of the oligomer. The second peak, which is also the most intense feature in both the spectra, corresponds to the $1 B_{3 u}^{+}$state. The dipole forbidden $1 B_{3 u}^{-}$state has been obtained at higher energy than the $1 B_{2 u}^{+}$state ( $c f$. Tables VII and VIII), which is different as compared to naphthalene and anthracene, but in agreement with the results of other investigators $(c f$. Table (II).

The comparison of our calculated results for $1 B_{3 u}^{-}, 1 B_{2 u}^{+}$, and $1 B_{3 u}^{+}$excited states with the several experimental and other calculated results is present in Table II. Our standard parameter results generally overestimate the excitation energies when compared to the experiments, while the agreement between the screened parameters results and the experiments $11,12,30,31,32$ is much better.

The $1 B_{2 u}^{+}$excitation energies computed using both the standard as well as the screened parameters overestimate all the experimental results. $11,12,29,30,31,32$ Yet our screened parameter value of the excitation energy of $1 B_{2 u}^{+}$state $(2.97 \mathrm{eV})$ is in reasonably good agreement with the experimental values of Klevens and Platt $(2.60 \mathrm{eV}), \frac{11}{1}$ Biermann et al. $(2.71 \mathrm{eV}), 12$ Bree and Lyons $(2.60 \mathrm{eV}), \frac{31}{\underline{ }} \operatorname{Birks}(2.63 \mathrm{eV}), \frac{32}{\underline{ }}$ and Berlman $(2.72 \mathrm{eV}) \stackrel{30}{\cdot}$ As far as the $1 B_{3 u}^{+}$ state is concerned, our screened parameter value of $4.38 \mathrm{eV}$ is fairly close to the several experimental values reported to be near $4.50 \mathrm{eV} ! \underline{11,12,31,32}$ However, our standard parameter value of $5.01 \mathrm{eV}$ for the same overestimates the experiments by about $0.5 \mathrm{eV}$. Our results for the dipole forbidden $1 B_{3 u}^{-}$state with both sets of parameters are in good agreement with the experiments. As compared to the experiments of Klevens and Platt 11 and Berlman ${ }^{30}$, our standard parameter excitation energy for the $1 B_{3 u}^{-}$is slightly higher, while the screened parameter value is slightly lower. Thus, we can conclude that in case of tetracene, for 
all the three excited states discussed, screened parameter based calculations provide better agreement with the experiments.

On comparing our results with other theoretical results we find that $1 B_{2 u}^{+}$and $1 B_{3 u}^{+}$states, computed using standard parameters are in excellent agreement with the benchmark work of Pariser, $\stackrel{44}{,}$ while the $1 B_{3 u}^{-}$state has been computed at lower value by both of our parameters. Ramasesha and co-workers also computed the optical gap of tetracene using P-P-P model Hamiltonian coupled with the DMRG technique.$^{50}$ They reported the $1 B_{2 u}^{+}$state at 3.20 $\mathrm{eV}$, which is in perfect agreement with the $1 B_{2 u}^{+}$state computed by us using the standard parameters. The results obtained using screened parameters for all the compared states are in very good agreement with the results of Ham et al. $\stackrel{46}{ }$ and with the ab initio MRMP based results of Kawashima et al. $\stackrel{48}{\underline{n}}$ The results of Rubio and coworkers ${ }^{51}$ obtained using B3LYP as xc functional for the $x$-polarized states $1 B_{3 u}^{-}$and $1 B_{3 u}^{+}$are higher than our results of the screened parameters, while is at low energy for the $y$-polarized $1 B_{2 u}^{+}$state.

\section{Pentacene}

The most thoroughly studied and most widely used member of the polyacene family is pentacene. It has been quite famous in recent years due to its use in thin film growth of electronic devices. ${ }^{81,82,83}$ It is among the most promising organic molecular semiconductor due to its high charge carrier mobility. $1,2,3$ It has been studied by several researchers to understand the nature of its low-lying excited states. However, most of the experimentalists have mainly reported the energy corresponding to the $1 B_{2 u}^{+}$state. ${ }^{29,34,35,37,38,39,40,41}$ As per our knowledge, experimentally only Klevens and Platt, 11 Biermann et al., $\frac{12}{1}$ and Birks ${ }^{32}$ have performed extensive studies of the $1 B_{3 u}^{-}, 1 B_{2 u}^{+}$, and $1 B_{3 u}^{+}$excited states. Additionally, Halasinski et al. $\frac{36}{n}$ measured the vibronic transitions of neutral pentacene isolated in Ne, Ar, and $\mathrm{Kr}$ matrices and reported the data corresponding to the $1 B_{3 u}^{-}, 1 B_{2 u}^{+}$, and $1 B_{3 u}^{+}$excited states. 


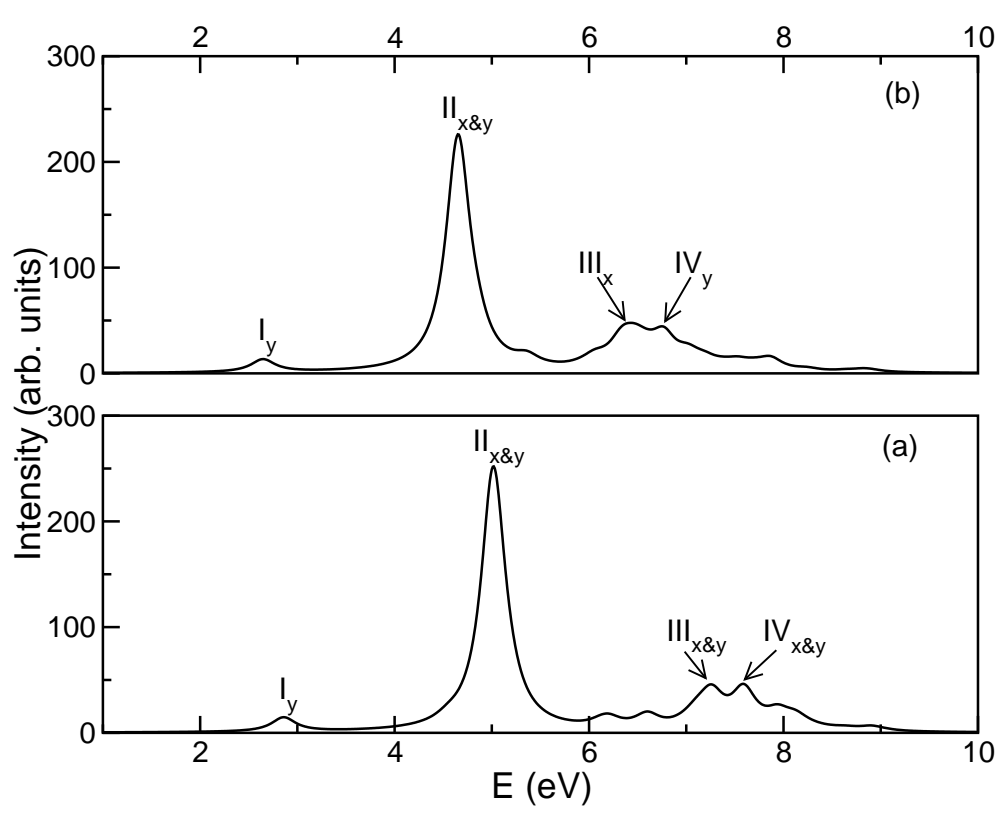

Figure 7: Linear optical absorption spectrum of pentacene computed with (a) standard parameters and (b) screened parameters. QCI method was used to compute the $B_{2 u}^{+}$states, while MRSDCI method was used to compute the $B_{3 u}^{+}$states.

Theoretically, the low-lying states of pentacene have been studied by several people, using various methodologies. $.44,46,50,56,57,84$ Similar to tetracene, for pentacene also we used the QCI method for computing the ground state $\left(1 A_{g}\right)$ and the $B_{2 u}$-type excited states, while the $B_{3 u}$-type excited states were computed using the MRSDCI technique.

In Fig:7 we present the linear optical absorption spectra computed using both standard and screened parameters in the P-P-P model Hamiltonian. The energies and wave functions corresponding to the visible features in the spectra as well as $1 B_{3 u}^{-}$state, are presented in the Tables $\mathrm{IX}$ and $\mathrm{X}$ in the appendix.

The first peak as usual, corresponds mainly to the $1 B_{2 u}^{+}$state, while the second peak is a mixture of states corresponding to $x$-polarized and $y$-polarized photons. But, as is clear from the transition dipoles, the intensity of the peak is mainly due to $x$-polarized photon corresponding to $H \rightarrow L+2+$ c.c. excitations leading to the $1 B_{3 u}^{+}$state (see Tables $\llbracket$ and X].

The comparison of our results with other theoretical and experimental results is presented in the Table II. On comparing our results with the experimental results we found that on most of the occasions both sets of parameters overestimate the experimental results. 
However, overall, the results obtained using the screened parameters are in much better agreement with the experiments. For example, the screened parameter results on $1 B_{2 u}^{+}$ and $1 B_{3 u}^{+}$states are in very good agreement with the experiments of Klevens and Platt, 11 Biermann et al. $\stackrel{12}{\underline{12}}$ Birkss $\stackrel{32}{\underline{3}}$ and Halasinski et al..$^{\underline{36}}$ However, several experimentalists have reported that the $1 B_{2 u}^{+}$state lies between $1.7 \mathrm{eV}$ to $1.9 \mathrm{eV} \underline{37}, \underline{38}, \underline{39}, 40,41$ The difference in our calculated results and these experimental results could be due to the bulk effects, as most of these experiments were performed on bulk pentacene, thus possibly explaining substantially lower values of band gaps. We again emphasize that our calculations were performed on single molecule of pentacene, leading to comparatively larger excitation energies.

Next, we compare our results with the theoretical results of other authors. Our correlated results for $1 B_{2 u}^{+}$and $1 B_{3 u}^{+}$obtained using standard parameters are in very good agreement with Pariser $\underline{44}^{\underline{4}}$ while the energy corresponding to $1 B_{3 u}^{-}$state is obtained to be lower in our standard parameter calculations. Our standard parameter value of $1 B_{2 u}^{+}$excitation energy $(2.86 \mathrm{eV})$ is also in excellent agreement with the value $2.92 \mathrm{eV}$ obtained by Raghu et al. $\underline{\underline{50}}$ from their DMRG calculations. Our results obtained using screened parameters are in very good agreement with the results obtained by Ham et al $\underline{\underline{46}}$ They used free electron molecular orbitals (FE MO) theory to compute the energies of the low-lying excited states. From our screened parameter calculations, the energy obtained for the $1 B_{3 u}^{-}$excited state is in perfect

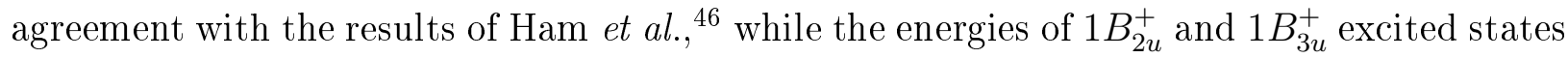
are slightly less than that of Ham et al. $\underline{\underline{46}}$ The energies of the $x$-polarized $1 B_{3 u}^{-}$and $1 B_{3 u}^{+}$ states obtained using standard as well as screened parameters agree well with the results for these states calculated by Rubio and coworkers, $\frac{51}{1}$ using xc functional. Recently, Tiago et $a l . \underline{\underline{4}}$ computed the energy gap for solution-phase crystallized (S) structure and vapor-phase crystallized (V) structure of pentacene using ab initio pseudo-potential density functional method and GW approach. They obtained the energy gap to be $2.2 \mathrm{eV}$ for $\mathrm{S}$ structure while $1.9 \mathrm{eV}$ for $\mathrm{V}$ structure. This low value of energy can be understood due to the bulk effects. Thus, their calculation supports our belief that bulk effects affect the $H \rightarrow L$ gap significantly. 


\section{Hexacene}

With the increase in the size of oligoacene, it becomes less stable, poorly soluble and more reactive.$\frac{85,86,87,88,89}{90}$ Thus, preparation and practical study of higher acenes like hexacene is a difficult task ${ }^{67}, 91$. To the best of our knowledge, experimentally hexacene has only been studied by Biermann $\underline{12}$ and Angliker et al..$\underline{42}$ They measured the absorption spectra of hexacene in solution. Angliker et al $\underline{\underline{42}}$ have also computed the low-lying excited states by performing P-P-P SCF-SCI calculations. Most recently, Grimme et al. $\frac{52}{\underline{5}}$ Heinze et al.,$\frac{54}{4}$ and Houk et al. $\stackrel{55}{\underline{5}}$ have also calculated the low-lying excited states using the time-dependent density functional theory (TDDFT) based approaches.

In Fig, [8, we present the linear absorption spectra of hexacene, computed using both standard and screened parameters in the P-P-P model Hamiltonian. For inclusion of electron correlation effects, the MRSDCI method was employed for all the states involved. The excited states, corresponding energies, transition dipoles, and wave functions of the various features of the spectra are presented in the Tables XI and XII in the appendix. Spectrum obtained using standard parameters predicts the $1 B_{2 u}^{+}$state at $2.71 \mathrm{eV}$, while from screened parameters it was obtained at $2.38 \mathrm{eV}$. In both the cases, the intensity of this peak (peak I) is quite small. In the standard parameter spectrum ( $c f$. Fig $8 \mathrm{a}$ ), the second peak corresponding to the to the $1 B_{3 u}^{+}$state is preceded by a weak shoulder $\left(\mathrm{II}_{y}\right)$ which is due to a $y$-polarized 
photon, and has been identified as $2 B_{2 u}^{+}$state.

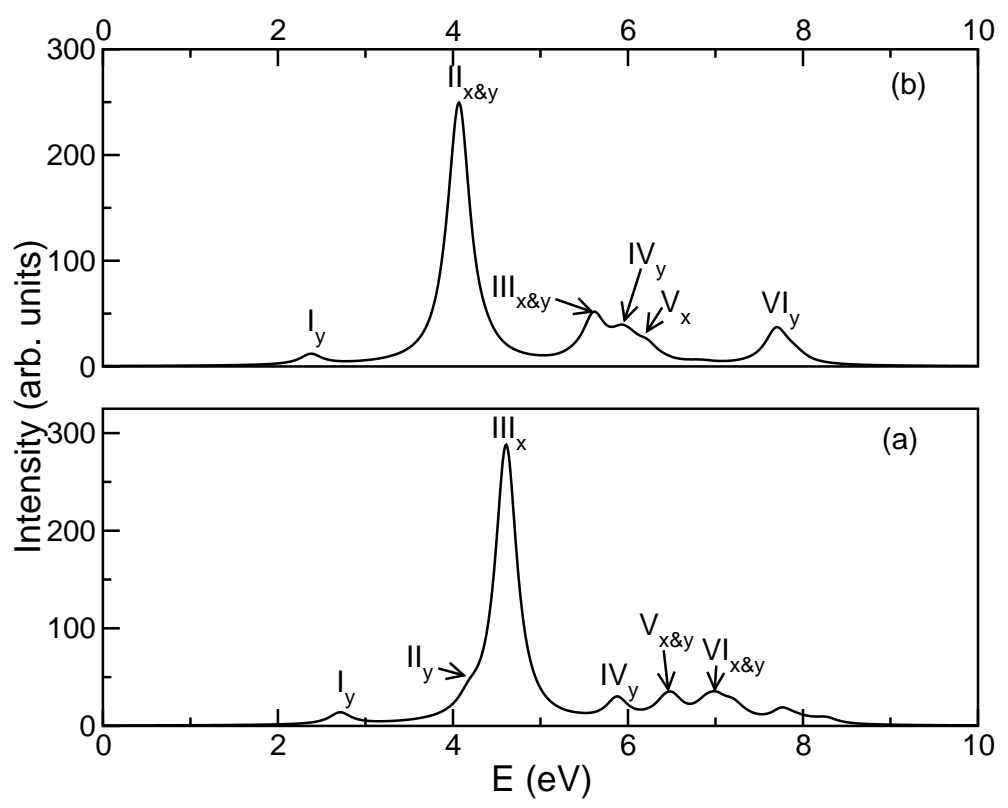

Figure 8: Linear optical absorption spectrum of hexacene computed with (a) standard parameters and (b) screened parameters. MRSDCI method was used to include the electron correlation effects.

In the screened parameter spectrum, this state is almost degenerate with $1 B_{3 u}^{+}$state and is part of the main feature II in the spectrum. However, with both sets of parameters, the intensity is mainly due to the $x$-polarized photon (see Tables XI and XII).

The remarkable feature of the dipole forbidden $1 B_{3 u}^{-}$state for hexacene is that its wave function mainly consists of double excitations, with the single excitations making smaller contributions. This is quite unlike the smaller acenes in which the $1 B_{3 u}^{-}$state consists mainly of singly excited configurations. Thus for this state, contribution of correlation effects appears to increase with size of the oligoacene.

Angliker et $a l .{ }^{42}$ have studied the spectra of hexacene experimentally as well as theoretically. They dissolved the hexacene in the silicone oil and measured the $1 B_{2 u}^{+}, 1 B_{3 u}^{-}$, and $1 B_{3 u}^{+}$ excited states at $1.91 \mathrm{eV}, 2.67 \mathrm{eV}$, and $3.94 \mathrm{eV}$ respectively. Theoretically they computed these states using P-P-P-SCF-SCI method at $2.18 \mathrm{eV}, 3.34 \mathrm{eV}$, and $4.05 \mathrm{eV}$ respectively. Our correlated calculations using standard parameters computed these states at $2.71 \mathrm{eV}$, $3.07 \mathrm{eV}$, and $4.61 \mathrm{eV}$ respectively, while screened parameters found these states to be at 2.38 $\mathrm{eV}, 2.77 \mathrm{eV}$, and $4.07 \mathrm{eV}$, respectively. Thus, generally our standard parameter calculations 
overestimate the experimental results of Angliker et al. $\underline{\underline{42}}$ for $1 B_{3 u}^{-}$state. However, with the screened parameters, our results are in good agreement with their experimental results 42 for the $1 B_{3 u}^{-}$and $1 B_{3 u}^{+}$excited states. Our screened parameter results for the $1 B_{2 u}^{+}$and the $1 B_{3 u}^{+}$states also agree well with their calculated results. $\underline{\underline{42}}$ Biermann et al $\underline{\underline{12}}$ observed $1 B_{3 u}^{-}$ at $2.80 \mathrm{eV}$ and $1 B_{2 u}^{+}$at $1.90 \mathrm{eV}$. Thus, in very good agreement with our screened parameter results for the first DF $x$-polarized state, while slightly overestimated for the first $y$-polarized state. Again, the trend is clear that the results obtained using the screened parameters are in better agreement with the experiments. $\underline{\underline{42}}$

Recently, Grimme and Parac ${ }^{\frac{52}{2}}$ used TDDFT and reported $1 B_{3 u}^{-}$and $1 B_{2 u}^{+}$states at 2.87 $\mathrm{eV}$ and $2.02 \mathrm{eV}$, which is in close agreement with our screened parameter results. Heinze et al $\frac{54}{\underline{4}}$ calculated the low-lying excited states of polyacenes using TDDFT based coupled Kohn-Sham (CKS) methodology. They report $1 B_{2 u}^{+}, 1 B_{3 u}^{+}$, and $1 B_{3 u}^{-}$states to be at 1.25 $\mathrm{eV}, 3.64 \mathrm{eV}$, and $2.76 \mathrm{eV}$, respectively. Rubio and coworkers ${ }^{51}$ reported these states to be at $1.50,3.94$, and $3.01 \mathrm{eV}$ respectively. Except for the $1 B_{2 u}^{+}$state, our results of screened parameters calculations are in very good agreement with their results. Additionally, Houk et al. $\underline{\underline{55}}$ computed the HOMO to LUMO gap ( $1 B_{2 u}^{+}$state in our notations) at $1.54 \mathrm{eV}$, using the TDDFT approach. The lower value of $1 B_{2 u}^{+}$excitation energy from the TDDFT calculations could be due to the usual problem of underestimating the band gaps, associated with the DFT based approaches.

\section{Heptacene}

The final member of the oligoacene family which we have studied is heptacene. It is a big molecule with seven diffused benzene rings, and $30 \pi$-electrons. It is an inherently unstable molecule,$\underline{92}$ whose synthesis has been under controversy $\underline{\underline{93}}$ Initially, Clar $\underline{\underline{94}}$ and Marschalk ${ }^{95}$ claimed the preparation of heptacene successfully. Later, Bailey and Liaio reported the only other successful synthesis of heptacene. $\underline{\underline{96}}$ Just after two years of the report of Bailey and Liaio, Clar retracted his earlier claim,,$\underline{\underline{94}}$ and now it is commonly agreed that heptacene cannot be prepared in the pure state. $\stackrel{97}{\underline{98}}$ Therefore, no experimental data is available till date for the low-lying excited states of heptacene. However, the low-lying excited states have been studied theoretically by a few researchers using the TDDFT method. $\stackrel{54,55}{5}$ 


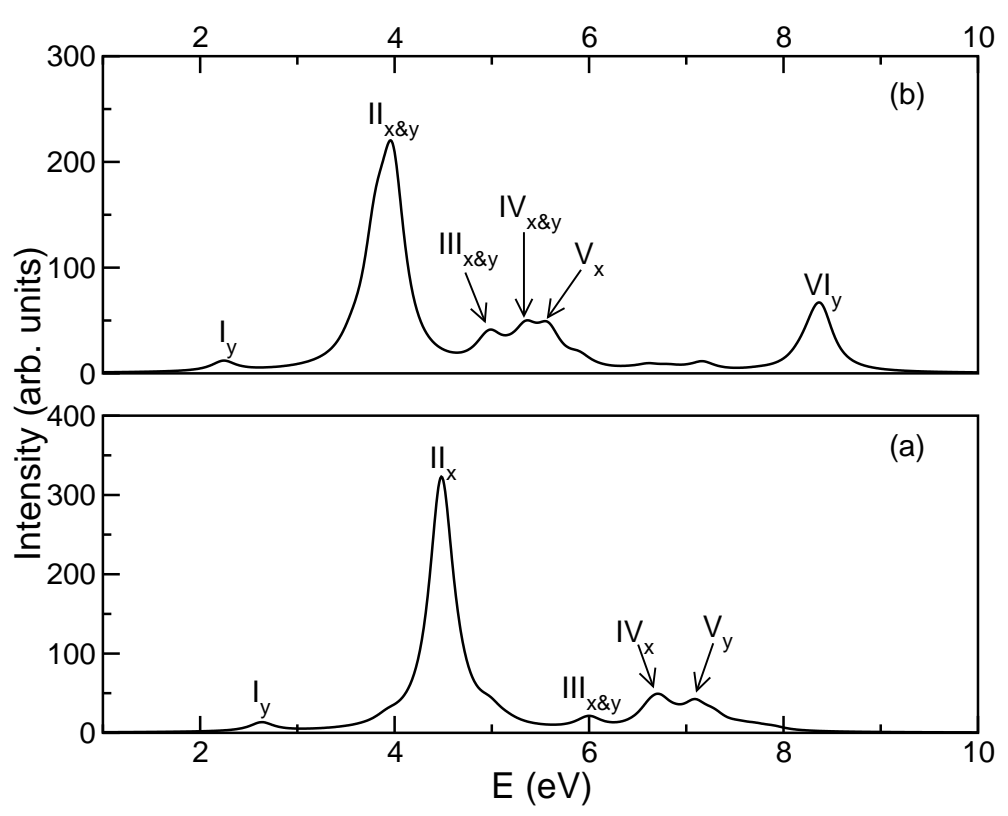

Figure 9: Linear optical absorption spectrum of heptacene computed with (a) standard parameters and (b) screened parameters. MRSDCI method, coupled with the P-P-P model Hamiltonian were used for the purpose.

We computed the linear absorption spectra of heptacene using standard and screened parameters in the P-P-P model Hamiltonian, together with the use of MRSDCI technique for the inclusion of the correlation effects. The calculated spectra are presented in Fig.9, while Tables XII] and XIV in the appendix provide the detailed information regarding the excited state properties. We note that, similar to the case of hexacene, the excited state wave functions of the $1 B_{3 u}^{+}$and $1 B_{3 u}^{-}$states are fundamentally different in that while the $1 B_{3 u}^{+}$consists mainly of one-particle excitations, while the $1 B_{3 u}^{-}$state is dominated by a double excitation, irrespective of the choice of the Coulomb parameters.

The first feature of Fig 9, corresponding to the $1 B_{2 u}^{+}$state, was obtained at $2.63 \mathrm{eV}$ from the standard parameter calculations, and at $2.24 \mathrm{eV}$ on using the screened parameters in the P-P-P Hamiltonian. The dipole forbidden $1 B_{3 u}^{-}$state was obtained at $2.73 \mathrm{eV}$ from the standard parameters, and at $2.35 \mathrm{eV}$ from the screened parameters. By both the parameters $1 B_{3 u}^{-}$is at higher energy than $1 B_{2 u}^{+}$, as is the case from tetracene onwards. Heinze et al. $\cdot \underline{\underline{54}}$ also calculated the excitation energies for heptacene using the TDDFT based CKS methodology $\underline{\underline{54}}$ They predicted $1 B_{2 u}^{+}$state at $0.943 \mathrm{eV}, 1 B_{3 u}^{-}$state at $2.349 \mathrm{eV}$ and $1 B_{3 u}^{+}$ state at $3.363 \mathrm{eV}$. Additionally, Houk et al.$\underline{55}$ used TDDFT method and computed the $1 B_{2 u}^{+}$ 
state (HOMO to LUMO gap) at $1.24 \mathrm{eV}$. Our $1 B_{2 u}^{+}$state is higher in comparison to both the DFT based results. Lower values of energy for $1 B_{2 u}^{+}$state obtained by DFT based methodology could be due to their well known problem of underestimating the band gaps. The dipole forbidden $1 B_{3 u}^{-}$states computed by us, using screened parameters is in perfect agreement with the results of Heinze et al.,$\frac{54}{\underline{w}}$ while the visible $1 B_{3 u}^{+}$state with maximum intensity is obtained at higher energies by both standard and screened parameters.

\section{CONCLUSIONS}

In this paper we presented a large-scale correlated study of linear optical absorption, and low-lying excited states, of oligoacenes ranging from naphthalene to heptacene, using the P-P-P model Hamiltonian. In order to investigate the influence of the Coulomb parameters on the computed properties, these calculations were performed with two sets of parameters in the P-P-P model: (i) standard Ohno parameters, $\underline{65}$ and (ii) a screened set of parameters. $\underline{66}$ An extensive comparison of our correlated results was made with: (i) the results obtained using the single-particle Hückel model, (ii) the experimental results, and (iii) the theoretical results of other authors. Next, in a unified manner, we present the conclusions which can be drawn from our calculations.

Qualitatively speaking, some aspects of the linear optical spectrum computed using the Hückel model, were found to be very similar to the one computed using the P-P-P model, for all the oligoacenes investigated. For example, with both sets of calculations, the first peak of the spectrum corresponded to the $1 B_{2 u}^{+}$state, while the second, and the most intense, peak of the spectrum corresponded to the $1 B_{3 u}^{+}$state for all the acenes. Both sets of calculations also predicted diminishing relative intensity of the $1 B_{2 u}^{+}$, with the increasing conjugation length. Additionally, irrespective of the parameters used in the P-P-P model, or the size of the acene involved, the many-particle wave function of the $1 B_{2 u}^{+}$state in all the cases consisted mainly of the $H \rightarrow L$ excitation, in excellent agreement with the Hückel model results. However, quantitatively speaking, for all the oligoacenes, the optical gaps computed using the Hückel model were much smaller than their P-P-P, and experimental, counterparts.

As far as the relative position of the dipole forbidden $1 B_{3 u}^{-}$excited state is concerned, with both the sets of Coulomb parameters the following trends were observed: (i) for oligoacenes up to anthracene, $1 B_{3 u}^{-}$occurs below the $1 B_{2 u}^{+}$state, however, (ii) from tetracene onwards the 
relative ordering of the two states gets reversed, and $1 B_{2 u}^{+}$state begins to occur below $1 B_{3 u}^{-}$. This theoretical result was found to be in good agreement with most of the experiments. Additionally, the influence of the electron correlation effects on $1 B_{3 u}^{-}$state appears to get stronger with the increasing size of the acene involved. This manifests itself in form of the trend that the contribution of the singly excited configurations to the many-particle wave function of $1 B_{3 u}^{-}$decreases with the increasing conjugation length, and is eventually overshadowed by the doubly-excited configurations for the larger acenes.

As mentioned earlier, for all the oligomers studied, irrespective of the Coulomb parameters used in the P-P-P Hamiltonian, $1 B_{3 u}^{+}$state was found to be the most intense peak of the spectrum, in excellent agreement with all the available experiments. Additionally, unlike $1 B_{3 u}^{-}$state, for all the acenes, the many-particle wave function of this most intense peak of the was dominated mainly by the singly-excited configurations. Although, with the increasing conjugation length, the relative contribution of the doubly-excited configurations to the $1 B_{3 u}^{+}$appears to increase. Therefore, it is conceivable that for conjugation lengths longer than the ones considered here, the contributions of the double excitations to $1 B_{3 u}^{+}$ could overshadow those of the single excitations. Yet, for a given conjugation length, the electron-correlation effects as judged from the relative contribution of the double excitations, appear to be stronger in case of $1 B_{3 u}^{-}$state, as compared to the $1 B_{3 u}^{+}$state.

Finally, we discuss the issue pertaining to the choice of Coulomb parameters for the PP-P model, when it comes to describing the linear optical properties of oligoacenes. We notice that in an overall sense, with the increasing conjugation length, the results obtained using the screened parameters are in a better quantitative agreement with the experiments as compared to those obtained using the standard parameters. There were some acenes for which both the parameters gave reasonable results, with the screened parameters energies slightly lower than the experimental energies, and the standard parameters ones slightly higher. Recalling that the screened parameters are generally used to describe the solidstate or solvent effects (interchain screening), the better quantitative agreement obtained using the screened parameters for the longer acenes, in our opinion, suggests the increasing importance of the solid-state effects on longer acenes.

In this paper we restricted ourselves to the low-lying excited states of polyacenes which contribute to their linear optical properties. However, it will also be interesting to explore the nature of their two-photon states, which will contribute to the nonlinear, as well as 
excited-state absorption, in these materials. Given the inherent anisotropy (short-axis vs. long-axis) of these materials, several types of intermediate states will possibly govern their nonlinear optical response. At present, studies along these directions are underway in our group.

\section{Acknowledgments}

We thank Department of Science and Technology (DST), Government of India, for providing financial support for this work under grant no. SP/S2/M-10/2000.

\section{Appendix A: EXCITED STATE PROPERTIES}

Here we present the tables summarizing the results of our CI calculations for various

oligoacenes. The data presented in the tables includes important configurations contributing to the many-body wave functions of various excited states, their excitation energies, and transition dipoles connecting them to the ground state. The results are presented in separate subsections corresponding to each oligoacene, and include calculations performed both with the standard parameters, and the screened set of parameters in the P-P-P model. 


\section{Naphthalene}

Table III: Excited states contributing to the linear absorption spectrum of naphthalene computed using the FCI method coupled with the standard parameters in the P-P-P model Hamiltonian. The table includes many particle wave functions, excitation energies, and dipole matrix elements of various states with respect to the ground state. DF corresponds to dipole forbidden state. '十c.c.' indicates that the coefficient of charge conjugate of a given configuration has the same sign, while '-c.c.' implies that the two coefficients have opposite signs.

\begin{tabular}{|c|c|c|c|c|}
\hline Peak & State & $\mathrm{E}(\mathrm{eV})$ & $\begin{array}{l}\text { Transition } \\
\text { Dipole }(\AA)\end{array}$ & Wave Functions \\
\hline DF & $1 B_{3 u}^{-}$ & 3.61 & 0.000 & $\begin{array}{c}|H \rightarrow L+1\rangle+\text { c.c. }(0.6235) \\
|H \rightarrow L+1 ; H-3 \rightarrow L\rangle+\text { c.c. }(0.1345) \\
|H-2 \rightarrow L+3\rangle+\text { c.c. }(0.1107)\end{array}$ \\
\hline I & $1 B_{2 u}^{+}$ & 4.45 & 0.551 & $\begin{aligned} \mid H & \rightarrow L\rangle(0.8773) \\
\mid H-1 & \rightarrow L+1\rangle(0.3521)\end{aligned}$ \\
\hline II & $\begin{array}{l}1 B_{3 u}^{+} \\
2 B_{2 u}^{+}\end{array}$ & 6.10 & 0.739 & $\begin{array}{c}|H \rightarrow L+1\rangle-\text { c.c. }(0.6524) \\
|H \rightarrow L+1 ; H-3 \rightarrow L\rangle-\text { c.c. }(0.1015) \\
|H-1 \rightarrow L+1\rangle(0.8011) \\
|H \rightarrow L\rangle(0.2958) \\
|H-2 \rightarrow L+2\rangle(0.2952)\end{array}$ \\
\hline III & $3 B_{2 u}^{+}$ & 7.78 & 1.028 & $\begin{array}{l}|H-2 \rightarrow L+2\rangle(0.8155) \\
|H-1 \rightarrow L+1\rangle(0.3002)\end{array}$ \\
\hline IV & $3 B_{3 u}^{+}$ & 8.73 & 0.281 & $\begin{array}{c}|H-2 \rightarrow L+3\rangle-\text { c.c. }(0.4965) \\
|H \rightarrow L+4\rangle-\text { c.c. }(0.2280) \\
|H \rightarrow L+1 ; H-1 \rightarrow L+2\rangle-\text { c.c. }(0.2260)\end{array}$ \\
\hline
\end{tabular}


Table IV: Excited states contributing to the linear absorption spectrum of naphthalene computed using the FCI method coupled with the screened parameters in the P-P-P model Hamiltonian. The table includes many particle wave functions, excitation energies, and dipole matrix elements of various states with respect to the ground state. DF corresponds to dipole forbidden state. '十c.c.' indicates that the coefficient of charge conjugate of a given configuration has the same sign, while '-c.c.' implies that the two coefficients have opposite signs.

\begin{tabular}{|c|c|c|c|c|}
\hline Peak & State & E $(\mathrm{eV})$ & $\begin{array}{r}\text { Transition } \\
\text { Dipole }(\AA)\end{array}$ & Wave Functions \\
\hline DF & $1 B_{3 u}^{-}$ & 3.22 & 0.000 & $|H \rightarrow L+1\rangle-$ c.c. $(0.5965)$ \\
$|H \rightarrow L+1 ; H-3 \rightarrow L\rangle+$ c.c. $(0.1564)$ \\
\hline I & $1 B_{2 u}^{+}$ & 4.51 & 0.719 & $|H \rightarrow L\rangle(0.9145)$ \\
\hline II & $1 B_{3 u}^{+}$ & 5.30 & 1.640 & $|H \rightarrow L+1\rangle+$ c.c. $(0.6466)$ \\
\hline III & $2 B_{2 u}^{+}$ & 6.12 & 0.842 & $|H-1 \rightarrow L+1\rangle(0.9012)$ \\
\hline IV & $3 B_{2 u}^{+}$ & 7.52 & 0.844 & $|H-2 \rightarrow L+2\rangle(0.8496)$ \\
\hline
\end{tabular}




\section{Anthracene}

Table V: Excited states contributing to the linear absorption spectrum of anthracene computed using the FCI method coupled with the standard parameters in the P-P-P model Hamiltonian. The table includes many particle wave functions, excitation energies, and dipole matrix elements of various states with respect to the ground state. DF corresponds to dipole forbidden state. '十c.c.' indicates that the coefficient of charge conjugate of a given configuration has the same sign, while '-c.c.' implies that the two coefficients have opposite signs.

\begin{tabular}{|c|c|c|c|c|}
\hline Peak & State & E $(\mathrm{eV})$ & $\begin{array}{c}\text { Transition } \\
\text { Dipole }(\AA)\end{array}$ & Wave Functions \\
\hline DF & $1 B_{3 u}^{-}$ & 3.25 & 0.000 & $\begin{array}{c}|H \rightarrow L+1\rangle-\text { c.c. }(0.5963) \\
|H \rightarrow L+1 ; H-3 \rightarrow L\rangle-\text { c.c. }(0.1329) \\
|H-2 \rightarrow L+3\rangle+\text { c.c. }(0.1268)\end{array}$ \\
\hline I & $1 B_{2 u}^{+}$ & 3.66 & 0.728 & $|H \rightarrow L\rangle(0.8894)$ \\
\hline II & $1 B_{3 u}^{+}$ & 5.34 & 2.040 & $|H-1 \rightarrow L+1\rangle(0.2097)$ \\
\hline III & $4 B_{2 u}^{+}$ & 6.94 & 1.069 & $|H-2 \rightarrow L+2\rangle(0.6612)$ \\
\hline IV & $3 B_{3 u}^{+}$ & 7.70 & 0.436 & $|H-1 \rightarrow L+1\rangle(0.4093)$ \\
\hline V & $7 B_{2 u}^{+}$ & 8.30 & 0.769 & $|H-4 \rightarrow+4\rangle-$ c.c. $(0.4831)$ \\
$\mid$ & & & $|H-1 \rightarrow L+5\rangle+$ c.c. $(0.2120)$ \\
\hline
\end{tabular}


Table VI: Excited states contributing to the linear absorption spectrum of anthracene computed using the FCI method coupled with the screened parameters in the P-P-P model Hamiltonian. The table includes many particle wave functions, excitation energies, and dipole matrix elements of various states with respect to the ground state. DF corresponds to dipole forbidden state. '十c.c.' indicates that the coefficient of charge conjugate of a given configuration has the same sign, while '-c.c.' implies that the two coefficients have opposite signs.

\begin{tabular}{|c|c|c|c|c|}
\hline Peak & State & $\mathrm{E}(\mathrm{eV})$ & $\begin{array}{l}\text { Transition } \\
\text { Dipole }(\AA)\end{array}$ & Wave Functions \\
\hline $\mathrm{DF}$ & $1 B_{3 u}^{-}$ & 2.91 & 0.000 & $\begin{array}{c}|H \rightarrow L+1\rangle-\text { c.c. }(0.5670) \\
H \rightarrow L+1 ; H-3 \rightarrow L\rangle+ \text { c.c. }(0.1500)\end{array}$ \\
\hline $\mathrm{I}$ & $1 B_{2 u}^{+}$ & 3.55 & 0.747 & $|H \rightarrow L\rangle(0.8890)$ \\
\hline II & $1 B_{3 u}^{+}$ & 4.64 & 2.019 & $|H \rightarrow L+1\rangle+$ c.c. $(0.6215)$ \\
\hline III & $\begin{array}{c}2 B_{2 u}^{+} \\
3 B_{2 u}^{+}\end{array}$ & 6.01 & 0.669 & $\begin{array}{c}|H-1 \rightarrow L+1\rangle(0.7680) \\
|H \rightarrow L+4\rangle-c . c .(0.2283) \\
|H \rightarrow L+4\rangle-\text { c.c. }(0.5024) \\
|H-1 \rightarrow L+1\rangle(0.3616) \\
|H-2 \rightarrow L+2\rangle(0.2987)\end{array}$ \\
\hline IV & $2 B_{3 u}^{+}$ & 6.63 & 0.519 & $\begin{array}{c}|H \rightarrow L+5\rangle+c . c .(0.3598) \\
|H \rightarrow L ; H \rightarrow L+2\rangle+c . c .(0.3170) \\
|H-2 \rightarrow L+3\rangle-\text { c.c. }(0.3161)\end{array}$ \\
\hline $\mathrm{V}$ & $7 B_{2 u}^{+}$ & 7.82 & 0.744 & $\begin{array}{c}|H-4 \rightarrow L+4\rangle(0.5739) \\
|H-3 \rightarrow L+3\rangle(0.3848) \\
|H \rightarrow L ; H-1 \rightarrow L+2\rangle-\text { c.c. }(0.2012)\end{array}$ \\
\hline
\end{tabular}




\section{Tetracene}

Table VII: Excited states contributing to the linear absorption spectrum of tetracene computed using the QCI method for $A_{g}$ and $B_{2 u}$ states, and the MRSDCI method for $B_{3 u}$ states, coupled with the standard parameters in the P-P-P model Hamiltonian. The table includes many particle wave functions, excitation energies, and dipole matrix elements of various states with respect to the ground state. DF corresponds to dipole forbidden state. '+c.c.' indicates that the coefficient of charge conjugate of a given configuration has the same sign, while '-c.c.' implies that the two coefficients have opposite signs.

\begin{tabular}{|c|c|c|c|c|}
\hline Peak & State & E $(\mathrm{eV})$ & $\begin{array}{c}\text { Transition } \\
\text { Dipole }(\AA)\end{array}$ & Wave Functions \\
\hline DF & $1 B_{3 u}^{-}$ & 3.22 & 0.000 & $|H \rightarrow L+2\rangle-$ c.c. $(0.5887)$ \\
$|H-1 \rightarrow L+4\rangle+c . c .(0.1395)$ \\
$\mid$
\end{tabular}


Table VIII: Excited states contributing to the linear absorption spectrum of tetracene computed using the QCI method for $A_{g}$ and $B_{2 u}$ states, and the MRSDCI method for $B_{3 u}$ states, coupled with the screened parameters in the P-P-P model Hamiltonian. The table includes many particle wave functions, excitation energies, and dipole matrix elements of various states with respect to the ground state. DF corresponds to dipole forbidden state. '+c.c.' indicates that the coefficient of charge conjugate of a given configuration has the same sign, while '-c.c.' implies that the two coefficients have opposite signs.

\begin{tabular}{|c|c|c|c|c|}
\hline Peak & State & $\mathrm{E}(\mathrm{eV})$ & $\begin{array}{l}\text { Transition } \\
\text { Dipole }(\AA)\end{array}$ & Wave Functions \\
\hline DF & $1 B_{3 u}^{-}$ & 3.02 & 0.000 & $\begin{array}{c}|H \rightarrow L+2\rangle-\text { c.c. }(0.5750) \\
|H-2 \rightarrow L ; H \rightarrow L+4\rangle-\text { c.c. }(0.1552)\end{array}$ \\
\hline $\mathrm{I}$ & $1 B_{2 u}^{+}$ & 2.97 & 0.799 & $|H \rightarrow L\rangle(0.8683)$ \\
\hline II & $1 B_{3 u}^{+}$ & 4.68 & 2.356 & $|H \rightarrow L+2\rangle+c . c .(0.6269)$ \\
\hline III & $3 B_{2 u}^{+}$ & 5.41 & 0.671 & $\begin{array}{l}|H-1 \rightarrow L+1\rangle(0.7657) \\
|H \rightarrow L+3\rangle-\text { c.c. }(0.2076)\end{array}$ \\
\hline IV & $\begin{array}{l}2 B_{3 u}^{+} \\
4 B_{2 u}^{+}\end{array}$ & 6.10 & 0.573 & $\begin{array}{c}|H \rightarrow L ; H \rightarrow L+1\rangle+\text { c.c. }(0.4998) \\
|H-4 \rightarrow L+1\rangle+\text { c.c. }(0.2119) \\
|H-1 \rightarrow L+1 ; H \rightarrow L+1\rangle+\text { c.c. }(0.2004) \\
|H-2 \rightarrow L+2\rangle(0.7957)\end{array}$ \\
\hline $\mathrm{V}$ & $9 B_{2 u}^{+}$ & 7.37 & 0.901 & $\begin{array}{c}|H-3 \rightarrow L+2\rangle-c . c .(0.3768) \\
|H \rightarrow L ; H \rightarrow L+1\rangle-c . c .(0.2426) \\
|H \rightarrow L ; H \rightarrow L+5\rangle+c . c .(0.2345) \\
|H-1 \rightarrow L+4\rangle+c . c .(0.2177) \\
|H-3 \rightarrow L+3\rangle(0.7722)\end{array}$ \\
\hline VI & $16 B_{2 u}^{+}$ & 8.44 & 0.650 & $\begin{array}{c}|H-5 \rightarrow L+5\rangle(0.6119) \\
|H \rightarrow L+1 ; H-4 \rightarrow L+1\rangle+\text { c.c. }(0.1790)\end{array}$ \\
\hline
\end{tabular}




\section{Pentacene}

Table IX: Excited states contributing to the linear absorption spectrum of pentacene computed using the QCI method for $A_{g}$ and $B_{2 u}$ states, and the MRSDCI method for $B_{3 u}$ states, coupled with the standard parameters in the P-P-P model Hamiltonian. The table includes many particle wave functions, excitation energies, and dipole matrix elements of various states with respect to the ground state. DF corresponds to dipole forbidden state. '+c.c.' indicates that the coefficient of charge conjugate of a given configuration has the same sign, while '-c.c.' implies that the two coefficients have opposite signs.

\begin{tabular}{|c|c|c|c|c|}
\hline Peak & State & $\mathrm{E}(\mathrm{eV})$ & $\begin{array}{l}\text { Transition } \\
\text { Dipole }(\AA)\end{array}$ & Wave Functions \\
\hline $\mathrm{DF}$ & $1 B_{3 u}^{-}$ & 3.17 & 0.000 & $\begin{array}{c}|H \rightarrow L+2\rangle+\text { c.c. }(0.5519) \\
|H \rightarrow L ; H \rightarrow L+1\rangle+\text { c.c. }(0.1439) \\
|H-1 \rightarrow L+4\rangle+\text { c.c. }(0.1341)\end{array}$ \\
\hline I & $1 B_{2 u}^{+}$ & 2.86 & 0.838 & $|H \rightarrow L\rangle(0.8568)$ \\
\hline II & $\begin{array}{c}1 B_{3 u}^{+} \\
3 B_{2 u}^{+}\end{array}$ & 5.16 & 2.721 & $\begin{array}{c}|H \rightarrow L+2\rangle-c . c .(0.5950) \\
|H-1 \rightarrow L+4\rangle-c . c .(0.1467) \\
|H-1 \rightarrow L+1\rangle(0.5108) \\
|H \rightarrow L+3\rangle+c . c .(0.3155) \\
|H-2 \rightarrow L+2\rangle(0.2668)\end{array}$ \\
\hline III & $5 B_{3 u}^{+}$ & 7.23 & 0.413 & $\begin{array}{c}|H-1 \rightarrow L+4\rangle-c . c .(0.3674) \\
|H \rightarrow L ; H \rightarrow L+5\rangle+c . c .(0.2329) \\
|H \rightarrow L ; H \rightarrow L+1\rangle-c . c .(0.1848) \\
|H-1 \rightarrow L+1 ; H \rightarrow L+1\rangle-c . c .(0.2762) \\
|H \rightarrow L ; H-1 \rightarrow L+3\rangle-c . c .(0.2266) \\
|H \rightarrow L+7\rangle+c . c .(0.2263) \\
|H-3 \rightarrow L+3\rangle(0.4121) \\
|H-6 \rightarrow L+3\rangle+c . c .(0.2475) \\
|H-4 \rightarrow L+4\rangle(0.2374)\end{array}$ \\
\hline IV & $12 B_{2 u}^{+}$ & 7.58 & 0.672 & $\begin{aligned} \mid H-3 & \rightarrow L+3\rangle(0.3294) \\
\mid H-2 & \rightarrow L+2\rangle(0.2682) \\
36 \quad \mid H-5 & \rightarrow L+5\rangle(0.2125) \\
\mid H-1 & \rightarrow L+5\rangle- \text { c.c. }(0.2060)\end{aligned}$ \\
\hline
\end{tabular}


Table X: Excited states contributing to the linear absorption spectrum of pentacene computed using the QCI method for $A_{g}$ and $B_{2 u}$ states, and the MRSDCI method for $B_{3 u}$ states, coupled with the screened parameters in the P-P-P model Hamiltonian. The table includes many particle wave functions, excitation energies, and dipole matrix elements of various states with respect to the ground state. DF corresponds to dipole forbidden state. '+c.c.' indicates that the coefficient of charge conjugate of a given configuration has the same sign, while '-c.c.' implies that the two coefficients have opposite signs.

\begin{tabular}{|c|c|c|c|c|}
\hline Peak & State & $\mathrm{E}(\mathrm{eV})$ & $\begin{array}{l}\text { Transition } \\
\text { Dipole }(\AA)\end{array}$ & Wave Functions \\
\hline $\mathrm{DF}$ & $1 B_{3 u}^{-}$ & 2.99 & 0.000 & $\begin{array}{c}|H \rightarrow L+2\rangle+\text { c.c. }(0.5117) \\
|H \rightarrow L ; H \rightarrow L+1\rangle-\text { c.c. }(0.2348) \\
|H \rightarrow L+2 ; H-4 \rightarrow L\rangle-\text { c.c. }(0.1394)\end{array}$ \\
\hline $\mathrm{I}$ & $1 B_{2 u}^{+}$ & 2.65 & 0.824 & $|H \rightarrow L\rangle(0.8464)$ \\
\hline II & $\begin{array}{l}1 B_{3 u}^{+} \\
2 B_{2 u}^{+}\end{array}$ & $\begin{array}{l}4.65 \\
4.63\end{array}$ & $\begin{array}{l}2.642 \\
0.317\end{array}$ & $\begin{array}{c}|H \rightarrow L+2\rangle-c . c .(0.6146) \\
|H \rightarrow L+3\rangle+c . c .(0.5123) \\
|H \rightarrow L\rangle(0.3994)\end{array}$ \\
\hline III & $4 B_{3 u}^{+}$ & 6.47 & 0.593 & $\begin{array}{c}|H-4 \rightarrow L+1\rangle-c . c .(0.4034) \\
|H-1 \rightarrow L+1 ; H \rightarrow L+1\rangle+c . c .(0.2226) \\
|H \rightarrow L ; H-1 \rightarrow L+3\rangle-c . c .(0.2216)\end{array}$ \\
\hline IV & $11 B_{2 u}^{+}$ & 6.77 & 0.782 & $\begin{array}{c}|H-3 \rightarrow L+3\rangle(0.7460) \\
|H \rightarrow L ; H \rightarrow L ; H-3 \rightarrow L+3\rangle(0.2030) \\
|H \rightarrow L+6\rangle-\text { c.c. }(0.1413)\end{array}$ \\
\hline
\end{tabular}




\section{Hexacene}

Table XI: Excited states contributing to the linear absorption spectrum of hexacene computed using the MRSDCI method, coupled with the standard parameters in the P-P-P model Hamiltonian. The table includes many-particle wave functions, excitation energies, and dipole matrix elements of various states with respect to the ground state. DF corresponds to dipole forbidden state. '十c.c.' indicates that the coefficient of charge conjugate of a given configuration has the same sign, while '-c.c.' implies that the two coefficients have opposite signs.

\begin{tabular}{|c|c|c|c|c|}
\hline Peak & State & $\mathrm{E}(\mathrm{eV})$ & $\begin{array}{l}\text { Transition } \\
\text { Dipole }(\AA)\end{array}$ & Wave Functions \\
\hline $\mathrm{DF}$ & $1 B_{3 u}^{-}$ & 3.07 & 0.000 & $\begin{array}{c}|H \rightarrow L ; H \rightarrow L+1\rangle+\text { c.c. }(0.4407) \\
|H \rightarrow L+3\rangle+\text { c.c. }(0.2729) \\
|H \rightarrow L+7\rangle+\text { c.c. }(0.1758)\end{array}$ \\
\hline I & $1 B_{2 u}^{+}$ & 2.71 & 0.812 & $|H \rightarrow L\rangle(0.8584)$ \\
\hline II & $2 B_{2 u}^{+}$ & 4.19 & 0.733 & $\begin{array}{c}|H \rightarrow L+2\rangle+\text { c.c. }(0.5045) \\
|H \rightarrow L ; H-1 \rightarrow L+3\rangle+\text { c.c. }(0.1810) \\
|H \rightarrow L ; H \rightarrow L+4\rangle-\text { c.c. }(0.1720)\end{array}$ \\
\hline III & $1 B_{3 u}^{+}$ & 4.61 & 3.049 & $\begin{array}{c}|H \rightarrow L+3\rangle-\text { c.c. }(0.5550) \\
|H-4 \rightarrow L+1\rangle+\text { c.c. }(0.1653)\end{array}$ \\
\hline IV & $5 B_{2 u}^{+}$ & 5.87 & 0.764 & $\begin{array}{c}|H \rightarrow L+6\rangle+\text { c.c. }(0.4457) \\
|H-1 \rightarrow L+5\rangle+\text { c.c. }(0.1821)\end{array}$ \\
\hline $\mathrm{V}$ & $\begin{array}{c}4 B_{3 u}^{+} \\
9 B_{2 u}^{+}\end{array}$ & 6.42 & 0.549 & $\begin{array}{c}|H \rightarrow L ; H \rightarrow L+1\rangle+\text { c.c. }(0.3513) \\
|H-1 \rightarrow L+4\rangle+\text { c.c. }(0.2390) \\
|H-1 \rightarrow L+1 ; H \rightarrow L+1\rangle-\text { c.c. }(0.2092) \\
\quad|H-1 \rightarrow L+5\rangle+\text { c.c. }(0.2787) \\
|H \rightarrow L+1 ; H \rightarrow L+3\rangle+\text { c.c. }(0.2784)\end{array}$ \\
\hline VI & $6 B_{3 u}^{+}$ & 6.90 & 0.256 & $\begin{array}{c}|H-3 \rightarrow L+3\rangle(0.4731) \\
|H-1 \rightarrow L+5\rangle+\text { c.c. }(0.2414) \\
|H-5 \rightarrow L+5\rangle(0.2177) \\
|H \rightarrow L ; H \rightarrow L+8\rangle+\text { c.c. }(0.4054) \\
|H \rightarrow L ; H-1 \rightarrow L+6\rangle+\text { c.c. }(0.2608) \\
|B 8 \rightarrow L ; H-2 \rightarrow L+5\rangle+\text { c.c. }(0.2090) \\
|H-1 \rightarrow L+4\rangle+\text { c.c. }(0.3445)\end{array}$ \\
\hline
\end{tabular}


Table XII: Excited states contributing to the linear absorption spectrum of hexacene computed using the MRSDCI method, coupled with the screened parameters in the P-P-P model Hamiltonian. The table includes many particle wave functions, excitation energies, and dipole matrix elements of various states with respect to the ground state. DF corresponds to dipole forbidden state. '十c.c.' indicates that the coefficient of charge conjugate of a given configuration has the same sign, while '-c.c.' implies that the two coefficients have opposite signs.

\begin{tabular}{|c|c|c|c|c|}
\hline Peak & State & $\mathrm{E}(\mathrm{eV})$ & $\begin{array}{l}\text { Transition } \\
\text { Dipole }(\AA)\end{array}$ & Wave Functions \\
\hline DF & $1 B_{3 u}^{-}$ & 2.77 & 0.000 & $\begin{array}{c}|H \rightarrow L ; H \rightarrow L+1\rangle+\text { c.c. }(0.4943) \\
|H \rightarrow L+3\rangle+\text { c.c. }(0.1830) \\
|H-1 \rightarrow L ; H \rightarrow L+2\rangle-\text { c.c. }(0.1819)\end{array}$ \\
\hline $\mathrm{I}$ & $1 B_{2 u}^{+}$ & 2.38 & 0.787 & $|H \rightarrow L\rangle(0.8683)$ \\
\hline II & $\begin{array}{c}2 B_{2 u}^{+} \\
1 B_{3 u}^{+}\end{array}$ & 4.07 & $\begin{array}{l}0.716 \\
2.948\end{array}$ & $\begin{array}{c}|H-1 \rightarrow L+1\rangle(0.7077) \\
|H \rightarrow L+2\rangle-\text { c.c. }(0.3154) \\
|H \rightarrow L+3\rangle-\text { c.c. }(0.5956)\end{array}$ \\
\hline III & $\begin{array}{c}6 B_{2 u}^{+} \\
4 B_{3 u}^{+}\end{array}$ & 5.61 & 0.414 & $\begin{array}{c}|H \rightarrow L+6\rangle+c . c .(0.3583) \\
|H-1 \rightarrow L+5\rangle-\text { c.c. }(0.3188) \\
|H-2 \rightarrow L+2\rangle(0.2851) \\
|H-1 \rightarrow L+4\rangle+\text { c.c. }(0.4188) \\
|H \rightarrow L+7\rangle-\text { c.c. }(0.2382) \\
|H \rightarrow L ; H-1 \rightarrow L+2\rangle+\text { c.c. }(0.2368) \\
|H-1 \rightarrow L+1 ; H \rightarrow L+1\rangle-\text { c.c. }(0.2054)\end{array}$ \\
\hline IV & $9 B_{2 u}^{+}$ & 5.93 & 0.672 & $\begin{array}{c}|H-2 \rightarrow L+2\rangle(0.6354) \\
|H-1 \rightarrow L+5\rangle-\text { c.c. }(0.2745)\end{array}$ \\
\hline $\mathrm{V}$ & $8 B_{3 u}^{+}$ & 6.23 & 0.555 & $\begin{array}{c}|H-2 \rightarrow L+3\rangle+\text { c.c. }(0.5539) \\
|H \rightarrow L+7\rangle-\text { c.c. }(0.1646) \\
|H \rightarrow L ; H-1 \rightarrow L+2\rangle+\text { c.c. }(0.1097)\end{array}$ \\
\hline VI & $18 B_{2 u}^{+}$ & 7.71 & 0.714 & $|H-3 \rightarrow L+3\rangle(0.6973)$ \\
\hline
\end{tabular}




\section{Heptacene}

Table XIII: Excited states contributing to the linear absorption spectrum of heptacene computed using the MRSDCI method, coupled with the standard parameters in the P-P-P model Hamiltonian. The table includes many particle wave functions, excitation energies, and dipole matrix elements of various states with respect to the ground state. DF corresponds to dipole forbidden state. '十c.c.' indicates that the coefficient of charge conjugate of a given configuration has the same sign, while '-c.c.' implies that the two coefficients have opposite signs.

\begin{tabular}{|c|c|c|c|c|}
\hline Peak & State & $\mathrm{E}(\mathrm{eV})$ & $\begin{array}{l}\text { Transition } \\
\text { Dipole }(\AA)\end{array}$ & Wave Functions \\
\hline DF & $1 B_{3 u}^{-}$ & 2.73 & 0.000 & $\begin{array}{c}|H \rightarrow L ; H \rightarrow L+1\rangle+\text { c.c. }(0.4896) \\
|H \rightarrow L+1 ; H-2 \rightarrow L\rangle+\text { c.c. }(0.1904) \\
|H \rightarrow L+1 ; H \rightarrow L+2\rangle+\text { c.c. }(0.1712)\end{array}$ \\
\hline $\mathrm{I}$ & $1 B_{2 u}^{+}$ & 2.63 & 0.793 & $|H \rightarrow L\rangle(0.8563)$ \\
\hline II & $1 B_{3 u}^{+}$ & 4.48 & 3.251 & $\begin{array}{c}|H \rightarrow L+3\rangle-\text { c.c. }(0.5033) \\
|H \rightarrow L ; H \rightarrow L+1\rangle-\text { c.c. }(0.2170) \\
|H-1 \rightarrow L+5\rangle+\text { c.c. }(0.1789)\end{array}$ \\
\hline III & $\begin{array}{c}6 B_{2 u}^{+} \\
4 B_{3 u}^{+}\end{array}$ & 6.01 & 0.326 & $\begin{array}{c}|H \rightarrow L ; H \rightarrow L+5\rangle-c . c .(0.3368) \\
|H \rightarrow L+1 ; H \rightarrow L+3\rangle+c . c .(0.3312) \\
\quad|H-1 \rightarrow L+1\rangle(0.1997) \\
|H \rightarrow L ; H-1 \rightarrow L+2\rangle-c . c .(0.4204) \\
|H-2 \rightarrow L+2 ; H \rightarrow L+1\rangle-c . c .(0.1781)\end{array}$ \\
\hline IV & $7 B_{3 u}^{+}$ & 6.70 & 0.624 & $\begin{array}{c}|H-1 \rightarrow L+5\rangle+\text { c.c. }(0.3258) \\
|H \rightarrow L ; H-1 \rightarrow L+2\rangle-\text { c.c. }(0.2972) \\
|H \rightarrow L ; H \rightarrow L+4\rangle+\text { c.c. }(0.2444)\end{array}$ \\
\hline $\mathrm{V}$ & $12 B_{2 u}^{+}$ & 7.09 & 0.736 & $\begin{array}{l}|H-2 \rightarrow L+2\rangle(0.3457) \\
|H-3 \rightarrow L+3\rangle(0.3312)\end{array}$ \\
\hline
\end{tabular}


Table XIV: Excited states contributing to the linear absorption spectrum of heptacene computed using the MRSDCI method, coupled with the screened parameters in the P-P-P model Hamiltonian. The table includes many particle wave functions, excitation energies, and dipole matrix elements of various states with respect to the ground state. DF corresponds to dipole forbidden state. '十c.c.' indicates that the coefficient of charge conjugate of a given configuration has the same sign, while '-c.c.' implies that the two coefficients have opposite signs.

\begin{tabular}{|c|c|c|c|c|}
\hline Peak & State & $\mathrm{E}(\mathrm{eV})$ & $\begin{array}{l}\text { Transition } \\
\text { Dipole }(\AA)\end{array}$ & Wave Functions \\
\hline $\mathrm{DF}$ & $1 B_{3 u}^{-}$ & 2.35 & 0.000 & $\begin{array}{c}|H \rightarrow L ; H \rightarrow L+1\rangle+\text { c.c. }(0.5062) \\
|H-1 \rightarrow L ; H \rightarrow L+2\rangle+\text { c.c. }(0.1942) \\
|H \rightarrow L+1 ; H \rightarrow L+2\rangle+\text { c.c. }(0.1648)\end{array}$ \\
\hline $\mathrm{I}$ & $1 B_{2 u}^{+}$ & 2.24 & 0.795 & $|H \rightarrow L\rangle(0.8675)$ \\
\hline II & $3 B_{2 u}^{+}$ & 3.98 & 2.553 & $\begin{array}{c}|H \rightarrow L ; H \rightarrow L+1\rangle-\text { c.c. }(0.4795) \\
|H-1 \rightarrow L+1 ; H \rightarrow L+1\rangle-\text { c.c. }(0.2362) \\
|H \rightarrow L+3\rangle-\text { c.c. }(0.1781) \\
|H \rightarrow L+2\rangle+\text { c.c. }(0.5254) \\
|H-1 \rightarrow L+1\rangle(0.4140) \\
|H \rightarrow L+3\rangle-\text { c.c. }(0.5710)\end{array}$ \\
\hline III & $4 B_{3 u}^{+}$ & 4.98 & 0.799 & $\begin{array}{c}|H-2 \rightarrow L+2\rangle+(0.4342) \\
|H-1 \rightarrow L+4\rangle+\text { c.c. }(0.4189) \\
|H \rightarrow L+6\rangle+\text { c.c. }(0.2618) \\
|H \rightarrow L ; H-1 \rightarrow L+2\rangle-\text { c.c. }(0.4219)\end{array}$ \\
\hline IV & $\begin{array}{l}5 B_{3 u}^{+} \\
7 B_{2 u}^{+}\end{array}$ & 5.34 & 0.668 & $\begin{array}{c}|H \rightarrow L+7\rangle-\text { c.c. }(0.4384) \\
|H \rightarrow L ; H-1 \rightarrow L+2\rangle-\text { c.c. }(0.2542) \\
|H-2 \rightarrow L+2\rangle(0.5520) \\
|H-1 \rightarrow L+4\rangle+\text { c.c. }(0.3475)\end{array}$ \\
\hline $\mathrm{V}$ & $7 B_{3 u}^{+}$ & 5.57 & 0.867 & $\begin{array}{c}|H-1 \rightarrow L+5\rangle+\text { c.c. }(0.4741) \\
|H \rightarrow L+7\rangle-\text { c.c. }(0.2927) \\
|H \rightarrow L ; H-1 \rightarrow L+2\rangle-\text { c.c. }(0.1582)\end{array}$ \\
\hline VI & $20 B_{2 u}^{+}$ & 8.39 & 0.871 & $|H-4 \rightarrow L+4\rangle(0.7058)$ \\
\hline
\end{tabular}


* Electronic address: psony@phy.iitb.ac.in, shukla@phy.iitb.ac.in

1 D. J. Gundlach, Y. Y. Lin, T. N. Jackson, S. F. Nelson, and D. G. Schlom, IEEE Electron Device Lett. 18, 87 (1997).

2 S. F. Nelson, Y. Y. Lin, D. J. Gundlach, and T. N. Jackson, Appl. Phys. Lett. 72, 1854 (1998).

3 V. Y. Butko, X. Chi, D. V. Lang, and A. P. Ramirez, Appl. Phys. Lett. 83, 4773 (2003).

4 K. Tanabe, K. Ohzeki, S. Nankai, and T. Yamabe, J. Phys. Chem. 41, 1069 (1983).

5 S. Kivelson and O. L. Chapman, Phys. Rev. B 28, 7236 (1983).

6 A. Hepp, H. Heil, W. Weise, M. Ahles, R. Schmechel, and H. Von Seggern, Phys. Rev. Lett. 91, $157406(2003)$.

7 K. Hummer, P. Puschnig, and C. Ambrosch-Draxl, Phys. Rev. B 67, 184105 (2003).

8 K. Hummer and C. Ambrosch-Draxl, Phys. Rev. B 71, 081202(R) (2005).

9 Y. C. Cheng, R. J. Silbey, D. A. da Silva Filho, J. P. Calbert, J. Cornil, and J. L. Brédas, J. Chem. Phys. 118, 3764 (2003).

10 See, e.g., E. Clar, Polycyclic Hydrocarbons, (Academic Press, London, 1964).

11 H. B. Klevens and J. R. Platt, J. Chem. Phys. 17, 470 (1949).

12 D. Biermann and W. Schmidt, J. Am. Chem. Soc. 102, 3163 (1980).

13 J. B. Birks, L.G. Christophorou, and R.H. Huebner, Nature 217, 809 (1968).

14 D. Bebelaar, Chem. Phys. 3, 205 (1974).

15 A. Bergman and J. Jortner, Chem. Phys. Lett. 26, 323 (1974).

16 R. H. Huebner, S. R. Mielczarek, and C. E. Kuyatt, Chem. Phys. Lett. 16, 464 (1972).

17 A. S. Aleksandrovsky, S. V. Karpov, S. A. Myslivets, A. K. Popov, and V. V. Slabko, J. Phys. B: At. Mol. Opt. Phys. 26, 2965 (1993).

18 N. Mikami and M. Ito, Chem. Phys. Lett. 31, 472 (1975).

19 B. Dick and G. Hohlneicher, Chem. Phys. Lett. 84, 471 (1981).

20 A. Bergman and J. Jortner, Chem. Phys. Lett. 15, 309 (1972).

21 R. P. Steiner and J. Michl, J. Am. Chem. Soc. 100, 6861 (1978).

22 W. R. Lambert, P. M. Felker, J. A. Syage, and A. H. Zewail, J. Chem. Phys. 81, 2195 (1984).

23 B. Dick and G. Hohlneicher, Chem. Phys. Lett. 83, 615 (1981).

24 L. E. Lyons and G. C. Morris, J. Mol. Spectrosc. 4, 480 (1960). 
25 K. F. Man, S. Trajmar, J. W. McConkey, J. M. Ratliff and M. Khakoo, J. Phys. B: At. Mol. Opt. Phys. 25, 5245 (1992).

26 L. Sebastian, G. Weiser, G. Peter, and H. Bässler, Chem. Phys. 75, 103 (1983).

27 J. Wolf and G. Holhneicher, Chem. Phys. 181, 185 (1994).

28 E. Sackmann and H. Wöhwald, J. Chem. Phys 58, 5407 (1973).

29 L. Sebastian, G. Weiser, and H. Bässler, Chem. Phys. 61, 125 (1981).

30 I. B. Berlman, Handbook of flourescence spectra of aromatic molecules, 2nd edn. (Academic Press, New York).

31 A. Bree and L. E. Lyons, J. Chem. Soc., 5206 (1960).

32 J. B. Birks, Photophysics of Aromatic Molecules (Wiley-Interscience, New York, 1970).

33 P. D. Burrow, J. A. Michejda, and K. D. Jordan, J. Chem. Phys. 86, 9 (1987).

34 S. C. Dahlberg and M. E. Musser, J. Chem. Phys. 71, 2806 (1979).

35 E. Heinecke, D. Hartmann, R. Müller, and A. Hese, J. Chem. Phys. 109, 906 (1998).

36 T. M. Halasinski, D.M. Hudgins, F. Salama, L. J. Alamandola, and T. Bally, J. Phys. Chem. A 104, 7484 (2000).

37 S. P. Park, S. S. Kim, J. H. Kim, C. N. Whang, and S. Im, Appl. Phys. Lett. 80, 2872 (2002).

38 S. S. Kim, S. P. Park, J. H. Kim, and S. Im, Thin Solid Films 420-421, 19 (2002).

39 J. Puigdollers, C. Voz, A. Orpella, I. Martin, M. Vetter, and R. Alcubilla, Thin Solid Films 427, 367 (2003).

40 R. He, I. Dujovne, L. Chen, Q. Miao, C. F. Hirjibehedin, A. Pinczuk, C. Nuckolls, C. Kloc, and A. Ron, Appl. Phys. Lett. 84, 987 (2004).

41 J. Lee, S. S. Kim, K. Kim, J. H. Kim, and S. Im, Appl. Phys. Lett. 84, 1701 (2004).

42 H. Angliker, E. Rommel, and J. Wirz, Chem. Phys. Lett. 87, 208 (1982).

43 C. A. Coulson, Proc. Phys. Soc. 60, 257 (1948).

44 J. Pariser, J. Chem. Phys. 24, 250 (1956).

45 J. R. Platt, J. Chem. Phys. 17, 484 (1949).

46 N. S. Ham and K. Ruedenberg, J. Chem. Phys. 25, 13 (1956).

47 O. C. Hofer and R. M. Hedges, Chem. Phys. Lett. 6, 67 (1970).

48 Y. Kawashima, T. Hashimoto, H. Nakano, and K. Hirao, Theo. Chem. Acc. 102, 49 (1999).

49 C. Raghu, Y. Anusooya Pati, and S. Ramasesha, Phys. Rev. B 65, 155204 (2002).

50 C. Raghu, Y. Anusooya Pati, and S. Ramasesha, Phys. Rev. B 66, 035116 (2002). 
51 E. S. Kadantsev, M. J. Stott, and Angel Rubio, J. Chem. Phys. 124, 134901 (2006).

52 S. Grimme and M. Parac, Tech. Rep. Ser. - I. A. E. A. 4, 292 (2003).

53 K. B. Wiberg, J. Org. Chem. 62, 5720 (1997).

54 H. H. Heinze, A. Görling, and N. Rösch, J. Chem. Phys. 113, 2088 (2000).

55 K. N. Houk, S. L. Patrick, and M. Nendel, J. Org. Chem. 66, 5517 (2001).

56 M. Baldo, A. Grassi, R. Pucci, and P. Tomasello, J. Chem. Phys. 77, 2438 (1982).

57 N. O. Lipari and C. B. Duke, J. Chem. Phys. 63, 1768 (1975).

58 See, e.g., D. Barieswyl, D. K. Campbell, and S. Mazumdar, in Conjugated Conducting Polymers, edited by H. Keiss (Springer-Verlag, Berlin, 1992), pp. 7-133.

59 H. Ghosh, A. Shukla, and S. Mazumdar, Phys. Rev. B 62, 12763 (2000).

60 A. Shukla, Phys. Rev. B 65, 125204 (2002).

61 A. Shukla, H. Ghosh, and S. Mazumdar, Phys. Rev. B 67, 245203 (2003).

62 A. Shukla, Chem. Phys. 300, 177 (2004).

63 A. Shukla, Phys. Rev. B 69, 165218 (2004).

64 P. Sony and A. Shukla, Phys. Rev. B 71, 165204 (2005).

65 K. Ohno, Theor. Chim. Acta 2, 219 (1964).

66 M. Chandross and S. Mazumdar, Phys. Rev. B 55, 1497 (1997).

67 See, e.g., R. B. Campbell and J. M. Robertson, Acta Cryst. 15, 289 (1962).

68 M. Dierksen and S. Grimme, J. Chem. Phys. 120, 3544 (2004).

69 M. S. Deleuze, L. Claes, E. S. Kryachko, and J.-P. Francois, J. Chem. Phys. 119, 3106 (2003).

70 J. Cioslowski, J. Chem. Phys. 98, 473 (1993).

71 M. A. Garcia-Bach, A. Peñaranda, and D. J. Klein, Phys. Rev. B 45, 10891 (1992).

72 T. A. Niehaus, M. Rohlfing, F. Della Sala, A. DiCarlo, and Th. Frauenheim, Phys. Rev. A 71, 022508 (2005).

73 S. Ramasesha and Z. G. Soos, Chem. Phys. 91, 35 (1984).

74 S. Ramasesha, D. S. Galvao, and Z. G. Soos, J. Phys. Chem. 97, 2823 (1993).

75 P. Sony and A. Shukla, Synth. Met. 155, 316 (2005).

76 P. Tavan and K. Schulten, J. Chem. Phys. 70, 5414 (1979).

77 H. E. Simmons, J. Chem. Phys. 40, 3554 (1964).

78 H. Nakatsuji, M. Komori, and O. Kitao, Chem. Phys. Lett. 142, 446 (1987).

79 T. Hashimoto, H. Nakano, and K. Hirao, J. Chem. Phys. 104, 6244 (1996). 
80 J. L. Brédas and G. B. Street, J. Phys. C: Solid State Phys. 18, L651 (1985).

81 R. A. Street, D. Knipp, and A. R. Völkel, Appl. Phys. Lett. 80, 1658 (2002).

${ }^{82}$ H. Klauk, D. J. Gundlach, J. A. Nichols, and T. N. Jackson, IEEE Trans. Electron Devices 46, $1258(1999)$.

83 H. Klauk, D. J. Gundlach, and T. N. Jackson, IEEE Electronic Device Lett. 20, 289 (1999).

84 M. L. Tiago, J. E. Northrup, and S. G. Louie, Phys. Rev. B 67, 115212 (2003).

85 E. Clar, Polycyclic Hydrocarbons, Academic Press, London, Vol. 1, 2 (1964); E. Clar, The Aromatic Sextet, Wiley, London (1972); A. Bjorseth, Ed. Handbook of Polycyclic Aromatic Hydrocarbons, Dekker, New York (1983); M. Cooke, A. J. Dennis, Eds. Polynuclear Aromatic Hydrocarbons: A Decade of Progress, Battelle Press, Columbus (1988); D. Biermann, W. J. Schmidt, J. Am. Chem. Soc. 102, 8163 (1980); R. G. Harvey, Polycyclic Aromatic Hydrocarbons, Wiley-VCH, New York (1997).

86 J. -I. Aihara, Phys. Chem. Chem. Phys. 1, 3193 (1999), J. -I. Aihara, J. Phys. Chem. A 103, 7487 (1999); J. -I. Aihara, J. Am. Chem. Soc. 99, 2048 (1977).

87 J. Sauer, R. Sustmann, Angew. Chem., Int. Ed. Engl. 18, 779 (1980); I. Fleming, Frontier Orbital and Organic Chemical Reactions, Wiley, Chichester (1976).

88 W. E. Bachmann and L. B. Scott, J. Am. Chem. Soc. 70, 1458 (1948); U. Girreser, D. Giuffrida, F. H. Kohnke, J. P. Mathias, D. Philip, and J. F. Stoddart, Pure Appl. Chem. 65, 119 (1993); Y. Muruta, N. Kato, K. Fujiwara, and K. Komatsu, J. Org. Chem. 64, 3483 (1999).

89 W. C. Herndon and Jr. M. L. Ellzey, J. Am. Chem. Soc. 96, 6631 (1974); W. C. Herndon, J. Chem. Soc., Chem. Commun., 817 (1977).

90 D. Z. Wang and A. Streitwieser, Theo. Chem. Acc. 102, 78 (1999).

91 E. Clar, Chem. Ber. 72B, 2137 (1939); C. Marschalk, Bull. Soc. Chim. 6, 1112 (1939).

92 Q. Xu, H. M. Duong, F. Wudl and Y. Yang, Appl. Phys. Lett. 85, 3357 (2004).

93 M. Bendikov, F. Wudl, and D. F. Perepichka, Chem. Rev. 104, 4891 (2004).

94 E. Clar, Chem. Ber. 75B, 1330 (1942).

95 See also, C. Marschalk, Bull. Soc. Chim. 10, 511 (1943).

96 W. J. Bailey and C. -W. Liaio, J. Am. Chem. Soc. 77, 992 (1955).

97 B. Boggiano and E. Clar, J. Chem. Soc., 2681 (1957).

98 R. Notario and J. -L. M. Abbound, J. Phys. Chem. A 102, 5290 (1998). 